\title{
Unterschiedliche Handlungslogiken in transdisziplinären und transformativen Forschungsprojekten - Welche Risikokulturen entwickeln sich daraus und wie lassen sie sich konstruktiv einbinden?
}

\author{
Monika Gonser, Jochen Eckart, Charlotte Eller, Katharina Köglberger, \\ Elke Häußler \& Felix M. Piontek
}

\section{$1 \quad$ Einleitung}

Transdisziplinäre (und transformative) ${ }^{1}$ Forschungsprojekte finden an institutionellen Schnittstellen gesellschaftlicher Teilsysteme statt, wie beispielsweise Wissenschaft, Recht, Politik, Wirtschaft sowie Zivilgesellschaft. Vertreter(innen) aus den verschiedenen Teilsystemen arbeiten und forschen gemeinsam. Ein transdisziplinäres Vorgehen setzt per se eine Zusammenarbeit von Vertreter(inne)n mindestens zweier verschiedener Teilsysteme voraus, wovon das eine immer das Teilsystem der Wissenschaft sein muss. Systemtheoretisch betrachtet (bspw. Luhmann 1984; Schimank 2007) folgen Vertreter(innen) der einzelnen Teilsysteme jeweils spezifischen Handlungslogiken. ${ }^{2}$ Durch die Zusammenarbeit in einem Projekt werden die unterschiedlichen Handlungslogiken der Teilsysteme spürbar. Eine solche Zusammenarbeit kann und soll Synergien erzeugen, sie kann jedoch auch zu Konflikten zwischen den Institutionen oder Akteuren der Teilsysteme führen, die es zu erkennen und zu beheben gilt.

Sowohl Synergien als auch Konflikte im Zuge der Zusammenarbeit unterscheiden sich in ihrer Intensität je nach Zusammensetzung des Projektteams. Zudem bringt ein transformativer Forschungsansatz mit seinem Anspruch, Veränderungs-

1 Die Autor(innen) möchten einen Beitrag zur Methodik transdisziplinärer Forschung leisten, die auch transformativ vorgehen kann, aber nicht muss. Diese doppelte Ansprache wird im Beitrag durch die Klammersetzung ,transdisziplinär (und transformativ)“" ausgedrückt.

2 Das Verhältnis von Teilsystem und Akteur wird in Kapitel 2 erklärt. 
prozesse anzustoßen, sowie der damit einhergehenden Notwendigkeit, meist experimentelle Anwendungen (z. B. Realexperimente ${ }^{3}$ ) in der Praxis durchzuführen, ein intensiviertes Aufeinandertreffen unterschiedlicher Teilsysteme mit sich. Denn die transformativen Aktivitäten beschränken sich oft nicht allein auf ein gesellschaftliches Teilsystem. Sie tangieren meist, und häufig auch gewollt, mehrere Teilsysteme. Um in einem Projekt eine hohe Synergie zu erzielen und Konflikte zu vermeiden bzw. zu lösen, empfiehlt sich eine vertiefte Analyse der Handlungslogiken der am Projekt beteiligten Teilsysteme. Durch den transdisziplinären Charakter eines Forschungsprojekts werden zudem auch unterschiedliche Definitionen, Wahrnehmungen und Einschätzungen von Risiken sowie unterschiedliche Bereitschaften, diese zu übernehmen, deutlich und bedeutsam. Diese sogenannten Risikokulturen, die sich je nach Handlungslogik des Teilsystems unterscheiden, werden in diesem Beitrag hergeleitet und erklärt.

Der Beitrag bietet einen Analyserahmen, der bei der Konzipierung von transdisziplinären (und transformativen) Forschungsprojekten helfen soll, die Synergien zwischen den Teilsystemen zu stärken und mögliche Konflikte frühzeitig zu erkennen. Vor Beginn eines Projekts können damit bereits potenzielle Konflikte je nach beteiligten Vertreter(inne)n der verschiedenen Teilsysteme abgeschätzt, mögliche Vorkehrungen getroffen und die nötige Transparenz innerhalb des Projektteams hergestellt werden. Ebenso kann die analytische Betrachtung auch bei bereits laufenden Forschungsprojekten helfen, Konflikte zu verstehen und $\mathrm{zu}$ lösen. Sollten während der Durchführung eines Projekts (unerwartete) Konflikte auftreten, kann dieser Analyserahmen eine Hilfestellung zur Selbstreflexion sein, indem er dazu beiträgt, dass die Handlungslogiken der beteiligten Vertreter(innen) der verschiedenen Teilsysteme nachvollziehbar werden. Für die Leser(innen) soll dieser Beitrag folglich eine Hilfestellung sowohl bei der Planung als auch während der Durchführung eines transdisziplinären (und transformativen) Forschungsprojekts sein.

Die analytische Aufarbeitung der Erfahrungen mehrerer Reallabore (BaWüLabs $^{4}$ ) und ein Quervergleich dieser Erfahrungen bilden die Basis für diesen Beitrag. Diese BaWü-Labs wurden in unterschiedlichen Kontexten realisiert und widmeten sich unterschiedlichen Forschungsthemen. Konflikte zwischen den Institutionen und Akteuren verschiedener Teilsysteme sowie Risiken und Chancen

3 Realexperimente sind Interventionen, die gemeinsam mit Akteuren aus der Praxis konzipiert und durchgeführt werden, und die im Rahmen eines Reallabors oder verwandten Forschungsformats durchgeführt und wissenschaftlich begleitet werden (s. Arnold und Piontek 2018, S. 146ff.).

4 Das Ministerium für Wissenschaft, Forschung und Kunst Baden-Württemberg fördert(e) seit 2015 in zwei Förderlinien 14 sogenannte Reallabore, die im Folgenden als ,BaWü-Labs' bezeichnet werden (s. auch http://www.reallabore-bw.de, zugegriffen am 20.05.2019). 
werden mit Beispielen aus diesen BaWü-Labs illustriert, und Lösungsansätze, die in den BaWü-Labs gewählt wurden, werden reflektiert. Ein Reallabor ist eines von vielen möglichen transdisziplinären (und transformativen) Forschungsformaten, und zwar eines, das gesellschaftswissenschaftliche Forschung anknüpfungsfähig für die Praxis macht, indem es Lösungswissen zu gesellschaftlichen Fragen durch häufig experimentelle Anwendungen erprobt und Faktoren einer potenziellen Übertragbarkeit reflektiert (z. B. Schneidewind und Singer-Brodowski 2014; Arnold und Piontek 2018). Die Beispiele im vorliegenden Text stammen aus den BaWü-Labs, da die Autor(inn)en in diesem Kontext gearbeitet haben. Der präsentierte Analyserahmen sowie die dargelegte Aufarbeitung der BaWü-Labs dürften jedoch für transdisziplinäre (und transformative) Forschungsprojekte im Allgemeinen gelten, eine Verengung auf die BaWü-Labs oder das Format Reallabor wurde deshalb bewusst nicht vorgenommen.

Im Folgenden werden zunächst der Analyserahmen, der zur Entfaltung der Handlungslogiken verwendet wird, dargestellt und der Begriff Risiko eingeführt. Für die Teilsysteme Wissenschaft, Politik, Recht, Wirtschaft und Zivilgesellschaft werden dann unter anderem deren Funktionen, Leitwerte, Leitdifferenzen, Entscheidungsstrukturen, Finanzierungen sowie Risikoverständnis und Risikomanagement erklärt. Die aus den Handlungslogiken resultierenden Risikokulturen und damit einhergehende Differenzen bezüglich der Wahrnehmung von Risiken und der Bereitschaft, diese einzugehen, werden dargestellt. Ebenso werden das Verständnis von und der Umgang mit Misserfolgen erläutert. In der transdisziplinären Realität ergeben sich häufig spezifische Chancen und Konflikte aus dem Zusammentreffen der partizipierenden Vertreter(innen) der Teilsysteme. Diese werden mithilfe von Beispielen aus den BaWü-Labs kurz diskutiert. Die in den BaWü-Labs gewählten Lösungsansätze dienen den Leser(inne)n als Anregung bei der Durchführung des eigenen transdisziplinären (und transformativen) Forschungsprojekts.

\section{Analyserahmen}

Um die unterschiedlichen Kontexte von Akteuren in solchen Forschungsprojekten $\mathrm{zu}$ verdeutlichen und damit diskutierbar zu machen, wird an dieser Stelle das systemtheoretische Konzept der Handlungslogiken von funktional definierten gesellschaftlichen Teilsystemen eingeführt. Anschließend wird der Analyserahmen der Risikokultur dargestellt. Obwohl in der Alltagskommunikation und in der Wissenschaft oft verwendet, gibt es kein allgemein gültiges Verständnis, wie der Begriff Risiko definiert und worauf er angewendet wird, dies variiert je nach Disziplin, gesellschaftlichem Teilsystem und sozialem Kontext (Weißensteiner 2014). Nach Fischer (2016) ist Risiko ein „,contested concept“", welches zwischen den Disziplinen sowie teilweise auch innerhalb der Disziplinen unterschiedlich definiert wird. Die darüber hinaus auch in der Anwendung vielfältige Begriffs- 
auffassung erklären Bechmann und Stehr (2000) damit, dass Risiko ein soziales Konstrukt ist, das abhängig vom sozialen Kontext unterschiedliche Anwendungen findet. Auch Fischer (2016) sowie Rayner (1992) gehen davon aus, dass Risiken sozial vermittelt sind und nicht unabhängig vom gesellschaftlichen Kontext verstanden werden. Ausgehend vom Verständnis, dass Risiko sozial konstruiert ist, werden von individuellen und kollektiven Akteuren in transdisziplinären (und transformativen) Forschungsprojekten jeweils andere Definitionen von Risiko genutzt und der Begriff unterschiedlich angewendet.

\subsection{Was wird unter Teilsystemen und Handlungslogiken verstanden?}

Systemtheoretische Ansätze gehen in Anlehnung an Luhmann (1984) von einer funktionalen Differenzierung von Gesellschaften aus (beispielsweise Baecker 2016; Reichel 2012; Schimank 2012). In diesem Ausdifferenzierungsprozess bilden sich in Abhängigkeit von der je spezifisch zu erfüllenden Funktion arbeitsteilig vorgehende Teilsysteme heraus. $\mathrm{Zu}$ diesen Teilsystemen gehören etwa das politische System, das Rechtssystem, das Wissenschaftssystem oder das Wirtschaftssystem. Diese funktionieren selbstreferenziell, das heißt, sie beziehen sich in ihrer Handlungslogik einzig auf sich selbst. Luhmann analysiert dabei ausschließlich Kommunikation, also die Frage, wo ein Austausch von Informationen stattfindet und anschlussfähig ist und wo nicht. Individuen - und somit auch Akteure - kommen in seiner Analyseperspektive nicht vor. Der vorliegende Beitrag nimmt jedoch keine Analyseperspektive im strengen Luhmann'schen Sinne ein. Vielmehr folgt er Schimank, der aufbauend auf Luhmann bezüglich der Teilsysteme auch die in ihnen handelnden Akteure thematisiert. Schimank erklärt diese Ausweitung damit, dass die funktionale Ausdifferenzierung einer Gesellschaft auf lange Sicht nur dann erhalten bleibt, wenn es in der Gesellschaft im ausreichenden Maß Akteure gibt, die sich die jeweils verschiedenen Handlungslogiken zu eigen machen und ihre jeweilige Aufrechterhaltung unterstützen (Schimank 2007). ${ }^{5}$

5 Schimank erläutert das Eingebundensein in Teilsysteme und die darin vorhandenen unterschiedlichen Akteursebenen folgendermaßen: „Aus der Sicht eines individuellen Gesellschaftsmitglieds heißt funktionale Differenzierung: All sein Streben, Denken und Handeln wird je nach Ausprägung seines Inklusionsprofils, also seines Einbezugs in die verschiedenen Teilsysteme, von etwa einem Dutzend Leitsternen gelenkt - faktisch sind es in den allermeisten Fällen nicht mehr als ein halbes Dutzend, die das Gros der Lebensführung nachhaltig prägen [...]. Für Organisationen als zweiten Typ von Akteur in der modernen Gesellschaft gilt das noch viel zugespitzter. Während Individuen durch multiple Partialinklusion in einer Mehrzahl von Teilsystemen charakterisiert sind, gehören die meisten Organisationen einem und nur einem Teilsystem an [...]. Unternehmen beispielsweise sind Wirtschaftsorganisationen und folgen als solche 
Die jeweilige Handlungslogik eines Teilsystems leitet sich aus einem nur für dieses Teilsystem gültigen Leitwert ab. Dieser Leitwert bringt „die fraglose oberste Orientierung allen Handelns" (Schimank 2007, S. 116) im betreffenden Teilsystem zum Ausdruck. Aus dem jeweiligen Leitwert wiederum ergibt sich die zugehörige Leitdifferenz, die als „Weichensteller“ (Schimank 2007, S. 116) dient. Die Leitdifferenz hat zwei Ausprägungen, beispielsweise für das Wissenschaftssystem ,wahr' und ,unwahr'. Nur die Kommunikationsinhalte, die im Wissenschaftssystem als (vorläufig), wahr ${ }^{`}$ angenommen werden, sind zur weiteren Erkenntnisproduktion anschlussfähig (Egner 2008). Mithilfe des Leitwerts wird die Arbeitsteilung in der Gesellschaft ausdifferenziert und ausbalanciert. Allerdings führt dies auch dazu, dass ,die Belange der anderen Teilsysteme nur höchst widerwillig berücksichtigt" werden (Schimank 2012, S. 114).

In Kapitel 3 werden die zentralen Eigenschaften der in den BaWü-Labs vertretenen Teilsysteme Wissenschaft, Politik, Recht, Wirtschaft und Zivilgesellschaft dargestellt. Abgeleitet aus dem systemtheoretischen Analyseansatz werden Funktion, Leitwert und Leitdifferenz des jeweiligen Teilsystems dargestellt. Zudem werden Qualitätssicherung, Entscheidungsstrukturen und Finanzierungen für die Teilsysteme kurz beschrieben. Diese zentralen Aspekte werden anhand folgender Leitfragen für jedes Teilsystem dargestellt:

- Was ist die Funktion des Teilsystems, an welchem Leitwert und an welcher Leitdifferenz orientiert es sich?

- Wie sieht Qualitätssicherung im jeweiligen Teilsystem aus?

- Wie werden Entscheidungen im jeweiligen Teilsystem getroffen?

- Wie gestalten sich die Finanzierungszusammenhänge des jeweiligen Teilsystems?

Abschließend wird die Relevanz der jeweiligen Handlungslogik für die Zusammenarbeit in transdisziplinären (und transformativen) Forschungsprojekten in Abgrenzung zu Handlungslogiken anderer Teilsysteme dargestellt.

dem binären Code dieses Teilsystems gleichsam monomanisch - Gesichtspunkte anderer Teilsysteme wie etwa rechtliche Regelungen oder wissenschaftliche Erkenntnisse tauchen, wie bereits erwähnt, immer nur als untergeordnete Randbedingungen der Orientierung an Zahlungsfähigkeit auf. [...] Nicht einzelne Akteure jeweils für sich, sondern erst deren handelndes Zusammenwirken erzeugt, im Wechselspiel mit handlungsprägenden Strukturen, soziale Dynamiken. Dieses handelnde Zusammenwirken kann zwar prinzipiell dauerhaft mäandern, ohne dass sich feste Konstellationsstrukturen spieltheoretisch als Gleichgewicht konzipierbar - herausbilden; der Regelfall, gerade im Rahmen engmaschiger teilsystemischer Programmstrukturen, sind jedoch dauerhafte, manchmal sehr langlebige Strukturmuster" (Schimank 2007, S. 208). 
Obwohl nach Schimank (2007) deutlich wird, dass Organisationen in ihrer Handlungslogik zumeist nur einem Teilsystem zuzuordnen sind, gibt es immer wieder auch solche, die mehrere Handlungslogiken in sich vereinen und damit einen hybriden Akteur darstellen. Im Kontext der BaWü-Labs traf dies vor allem auf Kommunen zu. Diese vereinen Aspekte sowohl der Handlungslogik des Teilsystems Recht als auch solche des Teilsystems Politik in sich, da sie sowohl die Rechtspflege zur Aufgabe haben als auch, über die kommunale Hierarchie, Gestaltungsaufgaben aus der Politik - beispielsweise durch den oder die (Ober-)Bürgermeister(in) oder den Gemeinderat - übertragen bekommen.

\subsection{Was wird unter Risiko und Risikokulturen verstanden?}

Wie eingangs des Kapitels dargelegt, ist die Auffassung von Risiko sozial konstruiert. Da die Akteure in transdisziplinären (und transformativen) Forschungsprojekten aus verschiedenen gesellschaftlichen Teilsystemen stammen, haben sie auch jeweils eine spezifische Auffassung von Risiko, die in solchen Projekten aufeinandertreffen. Für die Analyse der verschiedenen Risikoauffassungen wird auf das begriffliche Instrumentarium der Risikoforschung zurückgegriffen.

Vereinfacht können eine sozialwissenschaftliche und eine mathematische Definition von Risiko unterschieden werden. In den Ingenieurwissenschaften und der Versicherungswirtschaft wird ein mathematischer (technologischer und ökonomischer) Begriff von Risikoverwendet. So beschreibt die VDI-Richtlinie 4006 Risiko als die Kombination aus der Häufigkeit und der Auswirkungen eines zu einem Schaden führenden Ereignisses (VDI 4006 Blatt 1). Risiko ist so verstanden ein Produkt aus der Wahrscheinlichkeit und der Schadenshöhe eines Ereignisses. Damit verbunden ist eine mathematische Auffassung von Risiko, die versucht, das Risiko zu kalkulieren und damit zu managen sowie zu kontrollieren (Bonß 2011). Motel und Richter (2016) bieten eine sozialwissenschaftliche Definition von Risiko: alle potenziellen Bedrohungen für die Zielerreichung eines individuellen oder kollektiven Akteurs. In einer solchen Auffassung von Risiko werden auch die aus Sicht des mathematischen Verständnisses häufig paradoxen Entscheidungen von Akteuren, ein Risiko einzugehen, berücksichtigt (Bonß 2011). Das für beide Auffassungen von Risiko zentrale Verständnis von Schäden wird im Nachfolgenden dargestellt.

Aus der sozialwissenschaftlichen Auffassung von Risiko ergibt sich das Konzept der Risikokultur, das unterschiedlich definiert wird (z. B. Hoitsch et al. 2005; Fischer 2016; Jahner und Kremar 2005). So bezeichnet der Begriff Risikokultur ein Gerüst aus Praktiken und Deutungen, durch die Risiken analysiert und gemanagt werden. Bei der Analyse von Risikokulturen wird betrachtet, wie die Akteure das Risiko wahrnehmen, welche Haltung die Akteure zum Risiko haben und wie die Akteure mit dem Risiko umgehen (Jahner und Kremar 2005; Fischer 2016). 
Die für die spezifische Analyse der Risikokultur von Akteuren in transdisziplinären (und transformativen) Forschungsvorhaben wie Reallaboren relevanten Fragestellungen werden im Folgenden hergeleitet.

Die Akteure berücksichtigen unterschiedliche Phänomene als Schäden. Nach Auffassung der Versicherungswirtschaft sind Schäden unfreiwillige Einbußen, die ein Akteur an einem geschützten Gut oder Interesse erleidet (Möller und Bruck 1980). Aus Sicht des Risikomanagements versteht Link (2001) unter Schäden die von einem Akteur negativ bewerteten Folgen eines Ereignisses. Schäden sind somit alle Beeinträchtigungen von Zielen oder Gütern von Akteuren durch externe Ereignisse; diese Beeinträchtigungen können ökonomischer, rechtlicher oder politischer Natur sein. Ein Schaden kann also auch ein Misserfolg von transdisziplinären (und transformativen) Forschungsprojekten oder Teilen davon sein, wenn diese aus Sicht mindestens der Akteure eines Teilsystems, im Sinne der jeweiligen Handlungslogik, schlechte, enttäuschende oder negative Ergebnisse oder Konsequenzen haben.

Zudem ist zu unterscheiden, ob sich die Auffassung von Risiko allein auf Schäden konzentriert oder ob auch Chancen betrachtet werden. So fokussiert die Definition von Motel und Richter (2016) auf Schäden. Kromschröder (2003) unterscheidet hingegen beim Risiko eine negative Seite der Schäden, die durch ein Ereignis entstehen können, und eine positive Seite der Chance, ein Ziel zu erreichen. Im Kontext des Risikomanagements wird unter Chance ein ungewisses Ereignis beschrieben, das sich, sollte es eintreffen, positiv auf das Erreichen eines Ziels auswirkt (Link 2001). Eine Chance stellt, so gesehen, der Erfolg von transdisziplinären (und transformativen) Forschungsprojekten oder Teilen davon dar, wenn diese gemäß der Handlungslogik mindestens eines Teilsystems gut gelaufen sind. Im Kontext des Risikomanagements besteht häufig die Annahme, dass Chancen nicht ohne die Gefahr von Schäden zu haben sind. Folglich führt die Meidung von Schäden zu einem Verzicht auf Chancen (Kromschröder 2003). Für das Verständnis der Risikokultur verschiedener gesellschaftlicher Teilsysteme ist zu analysieren, welche Schäden nach deren Handlungslogik berücksichtigt werden, und ob die Akteure allein die Gefahr von Schäden oder auch die Abwägung von Schäden und Chancen berücksichtigen.

Bei der Analyse der Risikokultur ist zudem zu betrachten, welche Eintrittswahrscheinlichkeit bei Schäden und welche Schadenshöhe ein Akteur gewillt ist, einzugehen, um eine Chance der Zielerreichung wahrzunehmen (Jahner und Kremar 2005). Um die Risikokultur verschiedener gesellschaftlicher Teilsysteme zu analysieren, ist also die sich aus der Handlungslogik ergebende Risikoaffinität oder Risikoaversion zu beschreiben. Risikoaffinität besteht, wenn bei einer Auswahl die Alternative mit der höchsten erwarteten Zielerreichung bevorzugt wird, auch wenn damit höhere Risiken verbunden sind. Risikoaversion ist die Wahl der 
Alternativen mit dem geringeren Risiko hinsichtlich des Eintretens des Ereignisses bzw. des geringstmöglichen Schadens.

Die Risikokultur umfasst auch das Risikomanagement als die Steuerungsaufgabe der Identifikation, des Bewertens, der Steuerung sowie der Kommunikation von Risiken z. B. einer Organisation (DIN ISO 31000). Die Risikoidentifikation stellt die erste Phase des Risikomanagementprozesses dar. Mittels einer systematischen Identifikation werden mögliche Risiken festgehalten. Darauf aufbauend erfolgt die Risikobewertung, um anschließend Maßnahmen zur Risikosteuerung zu ergreifen (Weißensteiner 2014, S. 18ff.). Risikomanagement hat zum Ziel, die Handlungsfähigkeit von Akteuren gegenüber eventuellen Hindernissen und Krisen zu stärken (Schmitz und Wehrheim 2006). Dies kann auch Maßnahmen des Projektmanagements umfassen, soweit sich diese auf den Umgang mit Risiken beziehen. Das Risikomanagement von Akteuren hängt von der jeweiligen Risikokultur von Organisationen (im weiteren Sinne auch gesellschaftlichen Teilsystemen) ab (Douglas 1992).

Die identifizierten Fragen für die Analyse der Risikokultur der verschiedenen gesellschaftlichen Teilsysteme sind:

- Welche Schäden und Chancen werden im Teilsystem berücksichtigt?

- Wie risikoaffin oder risikoavers ist die Handlungslogik des Teilsystems?

- Welches Risikomanagement wird im Teilsystem üblicherweise betrieben?

Diese Fragen werden im folgenden Kapitel für die verschiedenen Teilsysteme ebenfalls beantwortet, wobei jeweils Bezug darauf genommen wird, wie sich die Risikokultur eines Teilsystems bezogen auf transdisziplinäre (und transformative) Forschungsprojekte manifestiert.

\section{Teilsystemspezifische Risikokulturen in transdisziplinären (und transformativen) Forschungsprojekten}

Im Folgenden wird analysiert, wie die durch die Handlungslogiken der gesellschaftlichen Teilsysteme geprägten Risikokulturen der Akteure deren Wahrnehmung und Handeln in Bezug auf Risiken in Reallaboren und anderen transdisziplinären (und transformativen) Forschungsprojekten leiten. Dabei werden die Teilsysteme Wissenschaft, Politik, Recht, Wirtschaft und Zivilgesellschaft berücksichtigt. Die Analyse orientiert sich an den aus dem Analyserahmen hergeleiteten Fragen (s. Kapitel 2). Nicht eingegangen wird auf Bewertungen von Risiken aufgrund von Persönlichkeitsstrukturen und anderen Handlungsvoraussetzungen, die sich aus der Person und nicht aus der Handlungslogik eines Teilsystems ergeben. 


\subsection{Wissenschaft}

Wissenschaft - Der Begriff der Wissenschaft bezieht sich hier zum einen auf Einrichtungen, die aufgrund ihrer gesellschaftlichen Aufgabe, ihrer Struktur und ihrer diskursiven Einbindung der Forschung zuzurechnen sind. Lehre hingegen wird im vorliegenden Zusammenhang ebenso wenig reflektiert wie Forschung, die überwiegend auf ein kommerzielles Ziel hin betrieben wird. Zum anderen bezieht sich der Begriff auf individuelle Gesellschaftsmitglieder, die aufgrund individueller formaler Qualifikation und institutioneller Anbindung an Wissenschaftseinrichtungen der Wissenschaft zugerechnet werden.

Funktion, Leitwert, Leitdifferenz: Nach Schimank (2012) übernimmt das Teilsystem Wissenschaft die Funktion des Wissenserwerbs und der Wissensreflexion einer Gesellschaft. Entsprechend richtet sich die wissenschaftliche Handlungslogik am Leitwert der Wissenschaft aus, dem Streben nach Wahrheit und Erkenntnis. Im wissenschaftlich forschenden Sinn wird anhand der im Teilsystem Wissenschaft zugrunde gelegten Leitdifferenz , wahr/unwahr' entschieden, welche Informationen für das Teilsystem anschlussfähig sind. ,Unwahr“ ist dabei als ,falsifizierbar' zu verstehen. Sowohl die Veröffentlichung, wahrer' als auch ,unwahrer' Informationen oder Interpretationen kann wissenschaftlich produktiv sein - beispielsweise, wenn sie anschließend widerlegt werden. Das heißt, ein in diesem Sinne unwahres, weil eine Hypothese falsifizierendes, aber auch ein die Hypothese nicht bestätigendes oder unerwartetes Ergebnis ist aus Sicht der Wissenschaft erstmal unproblematisch, weil es für den Erkenntnisgewinn trotzdem relevant ist, indem es wissenschaftliches Denken auf neue Wege leitet.

Qualitätssicherung: Im Teilsystem Wissenschaft hat sich ein System der Qualitätssicherung entwickelt, das im Wesentlichen in zwei Ansätzen zum Ausdruck kommt. Erstens wird versucht, über ein Ausbildungssystem mit klarer Hierarchie in Form unterschiedlicher akademischer Statusgruppen, Umfang und Qualität der erworbenen inhaltlichen und methodischen Kompetenzen abzubilden, um daraus ein weiteres Mitwirken am Teilsystem Wissenschaft abzuleiten. Zweitens werden über ein umfassendes System der Peer-Begutachtung und des Peer-Diskurses Forschungsprojekte und -ergebnisse vor- und nachbereitend reflektiert, eingeordnet, verworfen oder weiterentwickelt. Dadurch wird Vorgehensweisen und Ergebnissen die notwendige Qualität zugesprochen. Durch die Anzahl der Veröffentlichungen und Zitate in peer-reviewten Formaten kann die Anschlussfähigkeit und Verwertbarkeit des forschenden Handelns aus der Perspektive des Teilsystems Wissenschaft nachgewiesen werden.

Entscheidungsstrukturen: Was die hierarchische Strukturierung des Teilsystems Wissenschaft betrifft, so sind Führungspositionen und Entscheidungsmacht an akademische Erfolge und Qualitätssiegel geknüpft, mit dem Professorentitel als dem weitestreichenden Status- und Hierarchiemerkmal. Zuständigkeiten und 
Verantwortungsumfang sind üblicherweise von der akademischen Qualifikationsstufe abhängig. Daneben sind aber auch die Anzahl der Zitierungen und Publikationen, die Höhe eingeworbener Förderungen etc. Indikatoren akademischen Erfolgs. Führungs- und Arbeitsstrukturen in der Wissenschaft unterscheiden sich zum Beispiel in Abhängigkeit von der Disziplin. Verallgemeinernd lässt sich sagen, dass strategische und grundsätzliche inhaltliche Entscheidungen zumeist der professoralen Ebene zugeordnet werden, operativ-inhaltliche eher der akademischen, nicht-professoralen Ebene. Nicht-inhaltliche, also administrative $\mathrm{Zu}-$ ständigkeiten fallen häufig, aber nicht ausschließlich, der nicht-akademischen Ebene zu.

Finanzierung: Forschung wird üblicherweise aus zwei Quellen finanziert, die aus der gleichen Institution kommen können, aber nicht müssen. Die meisten Forschungseinrichtungen erhalten eine Grundförderung, aus der feste Stellen, Räume, Anlagen etc. finanziert werden und in deren Rahmen (nebst Lehre) auch Forschung betrieben wird. Der größere Anteil der Forschungsfinanzierung geht über Drittmittelförderungen ein, die die Akteure forschungsprojektbezogen einwerben müssen. Darüber hinaus gibt es auch „Inter-Systembeziehungen“ (Schimank 2007, S. 119) beispielsweise zum Teilsystem Wirtschaft oder dem Teilsystem Politik. Diese tragen - oft über Forschungsfinanzierungen - Nutzungserwartungen und Ergebniserwartungen an die Wissenschaft heran.

Schäden und Chancen: Ein Schaden entsteht für die Wissenschaft nur, wenn im Rahmen eines transdisziplinären (und transformativen) Forschungsprojekts kein neues Wissen gewonnen werden kann und daher keine Aussagen im Sinne der Leitdifferenz von ,wahr/unwahr' getroffen werden können. Wie bereits dargelegt, bildet es für die Wissenschaft keinen Schaden, wenn durch die Forschung in einem solchen Projekt nicht das erwartete Ergebnis erzeugt wird. Dies wird als Erkenntnisschritt akzeptiert. Ein Schaden entsteht damit vor allem, wenn beispielsweise ein geplantes Realexperiment nicht umgesetzt wird und in der Folge keinerlei Daten zum Untersuchungsgegenstand vorliegen. Das Risiko des Schadens, dass kein Wissen gewonnen werden kann, ist bei transdisziplinären (und transformativen) Forschungsprojekten aufgrund der zahlreichen externen Einflussfaktoren sowie Unwägbarkeiten von Forschung in der , realen Welt' höher als bei Forschung unter kontrollierten Bedingungen. Akteure aus der Wissenschaft nehmen jedoch das mit transdisziplinären (und transformativen) Forschungsprojekten verbundene höhere Risiko von Schäden in Kauf, da sie höhere Chancen sehen, bestimmte Wissensbestände zu gewinnen sowie die Ergebnisse der Forschung schneller und direkter zur Umsetzung zu bringen.

Im Reallabor für nachhaltige Mobilitätskultur (,,BaWü-Lab RNM“) wurden ursprünglich sechs verschiedene Realexperimente zur Durchführung ausgewählt (vgl. Puttrowait et al. 2018). Aus verschiedenen Gründen kam es bei zwei Realexperimenten schließlich doch nicht zur Umsetzung. Während bei einem Fall relativ frühzeitig ein 
Schlussstrich gezogen werden konnte, flossen im anderen Fall über einen längeren Zeitraum Ressourcen in die Bearbeitung des Experiments. Die durch das Nichtzustandekommen der Realexperimente freiwerdenden Ressourcen konnten zum einen für die Durchführung der übrigen Realexperimente eingesetzt werden, zum anderen konnten Forschungsdaten auf anderem Wege erhoben werden, wodurch eine teilweise Kompensation gelang.

Im Reallabor GO Karlsruhe - Partizipative Forschung für den Fußgängerverkehr (, BaWü-Lab GO Karlsruhe“) wurden zusammen mit Akteuren aus der Praxis zahlreiche mögliche Realexperimente zur Förderung des Fußverkehrs identifiziert. Von den zunächst angedachten Realexperimenten konnte im Verlauf des Reallabors jedoch nur ca. die Hälfte auch realisiert werden. Die anderen Realexperimente wurden in verschiedenen Planungsstadien abgebrochen. Verschiedene im BaWü-Lab beteiligte Wissenschaftler(innen) hatten daher den Schaden, dass sie Arbeit in die Planung von Realexperimenten investiert hatten, ohne dass dadurch Ergebnisse gewonnen wurden. Jedoch konnten andere geplante Realexperimente umgesetzt werden, so dass insgesamt das Projektziel des Erkenntnisgewinns zum Fußverkehr erreicht werden konnte. In der Gesamtbilanz wurde daher der Schaden durch einzelne abgebrochene Realexperimente durch den realisierten Erkenntnisgewinn des Gesamtvorhabens aufgewogen.

Risikoaversion und Risikoaffinität: Forschung als ein langfristiger und mit Unsicherheiten verbundener Prozess ist per se risikobehaftet (Hanebuth et al. 2015). Auch Huber (2013) stellt Risikobereitschaft als einen Kern von Wissenschaft heraus, die Fragen formuliert und Projekte verfolgt, deren Ausgang ungewiss ist. Die Handlungslogik des Teilsystems Wissenschaft setzt demnach generell eine gewisse Risikoaffinität der Akteure voraus. Allgemein bietet das Teilsystem Wissenschaft Rahmenbedingungen (wie z. B. einen weitgehenden Haftungsausschluss oder eine erfolgsunabhängige Vergütung), die es Wissenschaftlern erlauben, risikoaffin zu handeln (Hanebuth et al. 2015).

Im Reallabor Space Sharing - Nutzungsintensivierung des Gebäudebestands durch Mehrfachnutzung und dynamische Programmierung (,BaWü-Lab Space Sharing“) wurde die Innovation Space Sharing (für ausführliche Informationen s. RLSS 2018) an einem Standort im Betrieb getestet. Dabei gab es zu Beginn viele Unbekannte in unterschiedlichen Bereichen, mit denen umgegangen werden musste, die zum Teil aber auch Forschungsfragen waren, die im Laufe des Projekts geklärt werden sollten. Diese zwei Arten von Unbekannten konnten jedoch nicht isoliert betrachtet werden, sondern standen in Wechselwirkung zueinander. So hätten eine Fehlentscheidung oder ein negativer Verlauf in einem Bereich bewirken können, dass der Betrieb gar nicht hätte durchgeführt werden können. Folglich hätten auch die Forschungsfragen in den anderen Bereichen nicht bearbeitet werden können. Folgende Unbekannte stellten unter anderem zu Beginn ein Risiko dar: Standortwahl (,Welcher Standort ist für Space Sharing geeignet?“), Bedarf (,Wie groß ist die Nachfrage aus der Stadtbevölkerung nach Space Sharing?“), Art der Nutzungen („Für welche Nutzungen wird Space Sharing angefragt? Wie lassen sich diese kombinieren?“), bauliche Adaptionen (,Welche baulichen Adaptionen sind für Space Sharing notwendig? Lassen sich diese am gewählten Standort umsetzen?“). Diese Risiken in Kauf zu nehmen, setzte eine gewisse Risikobereitschaft voraus. 
Das Reallabor BUGA:log - Logistische Nahversorgung im urbanen Raum mit automatisierten Transporteinheiten im Rahmen der Bundesgartenschau 2019 mit Quartiersund Stadtteilentwicklung ,Neckarbogen“ (,,BaWü-Lab BUGA:log “) hätte ohne eine hohe Risikobereitschaft aller Akteure gar nicht umgesetzt werden können. Bereits der Bezugsrahmen des Reallabors, das autonome Fahren, ist per se mit einer hohen Unsicherheit behaftet. Dann gab es zum Zeitpunkt des Aufsetzens des Reallabors noch zahlreiche unbekannte Umgebungsparameter (,,die Stadt im Werden“ als Teil der Ausstellungskonzeption der Bundesgartenschau 2019). Verschiedene Risiken, die mit den technischen und rechtlichen Voraussetzungen des autonomen Fahrbetriebs in Zusammenhang stehen, konnten erst im Laufe des Projekts adressiert und gelöst werden. Dass dies so sein würde, war den Partnern des Reallabors vorab bewusst und wurde explizit als ein Teil des gemeinsamen Projekts definiert und gelebt. Um dieses Reallabor umzusetzen, mussten nicht nur die eher risikoaffinen Wissenschaftler(innen) hohe Risiken in Kauf nehmen, sondern auch die eher risikoaversen Akteure aus der Kommune.

Risikomanagement: Das Risikomanagement in Bezug auf den wissenschaftlichen Erkenntnisgewinn ist bei transdisziplinären (und transformativen) Forschungsprojekten vergleichbar mit Forschung im Allgemeinen und ist insbesondere auf die Wahrung der Leitdifferenz von ,wahr/unwahr' für die Bewertung der Ergebnisse ausgelegt. Das Risikomanagement erfolgt zum einen durch vorab im Forschungsdesign berücksichtigte alternative Forschungspfade, zum anderen durch die Orientierung an den Standards guter wissenschaftlicher Praxis. Überprüft wird Letzteres durch die oben beschriebenen Mechanismen zur Veröffentlichung der Ergebnisse und der Peer-Begutachtung. In transdisziplinären (und transformativen) Forschungsprojekten besteht jedoch das höhere Risiko, dass gewonnene Erkenntnisse aufgrund des neuen Forschungsformats durch einige Peers aus der Wissenschaft nicht anerkannt werden. Darüber hinaus müssen die Akteure der Wissenschaft in solchen Projekten das Risiko des Ausfallens von Forschungsschritten insbesondere in Realexperimenten und den damit verbundenen Ausfall oder die Beeinträchtigung des Erkenntnisgewinns managen. Um mit diesem Risiko umzugehen, nutzen die Akteure der Wissenschaft Risikomanagementstrategien aus anderen Teilsystemen wie zum Beispiel Strategien des Projektmanagements aus der Wirtschaft.

Im BaWü-Lab GO Karlsruhe wurden, um den Erfolg des Reallabors zu gewährleisten und das Risiko von Schäden zu reduzieren, zahlreiche Instrumente des Projektmanagements wie bspw. interne Projektgruppen, Beirat von externen Partnern oder Monitoring von Projektschritten eingesetzt. Diese Maßnahmen wurden zwar nicht als Risikomanagement bezeichnet, erfüllten aber auch diese Funktion. So bestand bei den geplanten Realexperimenten im öffentlichen Straßenraum ein hohes Risiko, dass diese bereits frühzeitig in der Planungsphase aufgrund externer Einflussfaktoren nicht umgesetzt würden, wodurch keinerlei Forschungsergebnisse hätten gewonnen werden können. Durch die enge Abstimmung mit den externen Partnern im Beirat wurde versucht, frühzeitig zu identifizieren und zu bewerten, für welche geplanten Realexperimente ein hohes Ausfallrisiko besteht. Als Risikosteuerungsmaßnahmen wurde 
entweder darauf hingewirkt, die externen Hindernisse für diese Realexperimente zu beseitigen, oder es wurde der Arbeitsaufwand für deren Planung reduziert, um den Verlust an Arbeitsaufwand zu verringern.

Das Energielabor Tübingen - Potenziale, Partizipation, Perspektiven (,,BaWü-Lab Energielabor Tübingen“) agierte unter dem Motto „Gemeinsam zur Energiewende“ und wollte die Stadtgesellschaft, v. a. die Bewohner(innen) bestimmter Quartiere, für die Partizipation an Maßnahmen des Reallabors gewinnen. Entsprechend anberaumte Veranstaltungen für alle Bürger(innen) fanden aber zu wenig Anklang, so dass weder von echter breiter Partizipation hätte gesprochen werden können noch wissenschaftlich brauchbare Erkenntnisse hätten erzeugt werden können. Die getroffene Maßnahme des Risikomanagements bestand darin, dass Abstand genommen wurde vom Ziel, die gesamte Bevölkerung anzusprechen. Darauf wurden dann „Wendepunkt-Projekte“ als Realexperimente entworfen, die sich spezifischer auf die bereits im Bereich Energiewende Aktiven richteten. Diese Art von Partizipation konnte dann erfolgreich vor Ort durchgeführt werden.

\subsection{Politik}

Politik - Der Begriff der Politik bezieht sich auf Einrichtungen, deren Aufgaben die politische Gestaltung und die Vertretung individueller Gesellschaftsmitglieder sind, also beispielsweise Parlamente und Regierungen, aber auch demokratisch legitimierte Interessenverbände auf den verschiedenen föderalen Ebenen. Entsprechend sind auch einzelne Politiker(innen) als Angehörige derartiger Einrichtungen der Politik zuzurechnen.

Funktion, Leitwert und Leitdifferenz: Nach Schimank (2007) ist die Funktion des Teilsystems Politik das Treffen belastbarer kollektiver Entscheidungen über die Repräsentation von Interessen und Werten durch gewählte Mandatsträger im demokratischen System. Unter Interessen sind hierbei handlungsstiftende, individuelle und kollektive Ziele und Bedürfnisse zu verstehen (Rudzio 2015). Werte wiederum sind hier dem Individuum übergeordnete, normenstiftende Ideen eines guten gesellschaftlichen Zusammenlebens (Joas 2005). Der Leitwert des Teilsystems Politik sind möglichst umfassende Gestaltungsmöglichkeiten gesellschaftlicher Lebensumstände. Die Leitdifferenz ist ,Macht/keine Macht.

Qualitätssicherung: In Demokratien muss sich der Gestaltungswille des Teilsystems Politik über Wahlen legitimieren und unterliegt somit auch einer Qualitätssicherung im Sinne der Wähler(innen). Politische Entscheidungsstrukturen sind von der Legitimation durch Wahlen abhängig. In einem weiter gefassten Sinne lassen sich auch Aspekte indirekter politischer Partizipation von Bürger(inne)n als Qualitätssicherung verstehen, beispielsweise wenn in politischen Gestaltungsprozessen über Jurys oder Beteiligungsräte qualitative Einschätzungen zu verschiedenen Gestaltungsoptionen eingeholt werden. 
Entscheidungsstrukturen: Im Teilsystem Politik gilt typischerweise ein repräsentativer Ansatz. Der Gestaltungswunsch wird also durch die Wähler(innen) an die Politik delegiert. Im Unterschied zur Zivilgesellschaft (s. Abschnitt 3.5) kann bei ausreichender Unterstützung durch die Mehrheit eine Gestaltung auch für die nicht zustimmende Minderheit vorgenommen werden. Die Handlungslogik der Politik unterscheidet sich von der Handlungslogik der Zivilgesellschaft insofern, als die Leitdifferenz ,Macht/keine Macht' über Repräsentation und Mehrheitsentscheid gewonnen werden muss und den Akteuren diese Legitimation ihres Handelns auch immer wieder entzogen werden kann. Die Frage des Machterhalts oder der Machtausweitung zwischen den Wahlen hängt jedoch nur bedingt vom Wahlergebnis selbst ab und kann sich zwischen den Wahlen auch schwankend aus Netzwerken, politischer Kommunikation, Informationszugriff, Koalitionen etc. ergeben. Als gesellschaftliche Erwartung wird jedoch der Wunsch an politische Repräsentant(inn)en und Einheiten herangetragen, dass sich Macht möglichst ausschließlich und transparent aus Wahlergebnissen ableitet und dieses auch nach der Wahl von Politiker(inne)n als Grundlage für inhaltliche Gewichtungen während der Legislaturperiode herangezogen wird. Mit welcher Reichweite dieser Legitimationsmechanismus in der Realität tatsächlich greift (Crouch 2008), geht über den vorliegenden Beitrag hinaus.

Finanzierung: Politische Strukturen sind üblicherweise so aufgebaut, dass der Wählerwille über das Haushaltsrecht auch die finanziellen Mittel verleiht, um politische Schwerpunktsetzungen vorzunehmen. Die Finanzierung erfolgt in Deutschland über Steuergelder von Land, Bund und der Europäischen Union. Das Teilsystem Politik wird aber auch durch Mittel finanziert, die sich nicht aus der staatlichen Steuerungsstruktur vor allem über Steuern ergeben. Deshalb spielen auch Spenden, Sponsoring von Unternehmen oder beispielsweise Stiftungsgelder insbesondere bei Modellprojekten eine Rolle und führen zu der Entstehung von bereits genannten „Inter-Systembeziehungen“ namentlich zum Teilsystem Wirtschaft. Entstehen daraus Überschneidungen zwischen den Leitzielen der so verbundenen Teilsysteme, können daraus Konflikte aufgrund von Widersprüchen zwischen den Leitwerten resultieren.

Schäden und Chancen: Das zentrale Risiko, das sich aus der Handlungslogik des Teilsystems Politik ergibt, besteht darin, dass eine vorgenommene Handlung die Macht verkleinern könnte. Die betrachteten Schäden beziehen sich weniger auf ein transdisziplinäres (und transformatives) Forschungsprojekt als Ganzes, sondern konzentrieren sich auf die in der ,realen Welt' umgesetzten und wahrnehmbaren Maßnahmen, insbesondere Realexperimente. Für das Teilsystem Politik ist damit insbesondere die Außenwirkung beispielsweise eines Reallabors von Relevanz. Für die Politik entstehen Schäden, wenn die darin umgesetzten Realexperimente zwar wie erwartet funktionieren, die Wirkungen jedoch politisch (z. B. von einem Teil der Wählerschaft) nicht gewollt sind. Wenn die Maßnahme nicht dem 
Wählerwillen als Machtlegitimation der Politik entspricht, ergibt sich ein Konflikt in der Leitdifferenz ,Macht/keine Macht ${ }^{\star}$. Ein Schaden aus Sicht der Politik kann zudem dann eintreten, wenn beispielsweise die in einem Reallabor umgesetzten Realexperimente nicht die gewünschten Ergebnisse erzielen. Wie ausgeführt kann in der Wissenschaft auch eine fehlende Bestätigung einer Hypothese zu einem akzeptierten Wissensgewinn führen. Im Teilsystem Politik dagegen werden nicht erfüllte Erwartungen und das Lernen aus Fehlern nur sehr eingeschränkt akzeptiert. Dabei ist aus der Handlungslogik der Politik nicht so sehr problematisch, dass die Gestaltung der gesellschaftlichen Lebensumstände nicht wie gewünscht gelingt, sondern vielmehr, dass dies den Schaden eines Machtverlusts bewirken kann. So werden Fehler entsprechend der Leitdifferenz ,Macht/keine Macht' von der politischen Gegenseite häufig zur Kritik genutzt. Dies kann Vorwürfe umfassen wie z. B., mit öffentlichen Geldern nicht verantwortlich umgegangen zu sein oder Externe dem Risiko von Schäden ausgesetzt zu haben. Jedoch werden von der Politik auch Chancen von transdisziplinären (und transformativen) Forschungsprojekten wahrgenommen. Diese liegen meist darin, dass sie helfen, die verfolgten Gestaltungsziele umzusetzen. Wie bereits erwähnt, spielte die Handlungslogik des Teilsystems Politik im Rahmen der BaWü-Labs insbesondere für die Akteure aus kommunalen Verwaltungen eine relevante Rolle.

In Heilbronn wurde das BaWü-Lab BUGA:log von der Verwaltung auch gezielt genutzt, um - auch über die Fragestellungen des Reallabors hinausgehend - den Dialog zwischen der Stadt und den Anbietern innovativer Lösungen im Bereich Mobilität und Logistik zu intensivieren. Dies wurde als Chance gesehen, auch in anderen Bereichen die Transformation der Stadt im Mobilitätsbereich voranzubringen.

Der 2012 beschlossene Verkehrsentwicklungsplan Karlsruhe formuliert als politisches Ziel die verstärkte Förderung des Fußverkehrs. Das BaWü-Lab GO Karlsruhe, welches ab 2015 ebenfalls dieses Ziel verfolgte, wurde daher von der Kommunalpolitik der Stadt Karlsruhe positiv aufgenommen. Es wurde als eine Chance gesehen, die bereits beschlossene politische Zielsetzung umzusetzen. Dafür wurde das Risiko von politisch nicht gewollten Teilergebnissen und damit verbundenen Kontroversen in der Kommunalpolitik eingegangen.

Risikoaversion und Risikoaffinität: Die Risikobereitschaft des Teilsystems Politik wird durch zwei gegensätzliche Kräfte beeinflusst. Einerseits verfolgt es langfristige Gestaltungsabsichten, die es erforderlich machen, zumindest partiell Neues zu wagen und dafür auch Risiken einzugehen. Andererseits kann sich Politik Misserfolge bei der Umsetzung ihrer Gestaltungsabsichten ohne erhebliche Nachteile durch Kritik seitens politischer Gegner nicht leisten. Die Politik wägt daher die Chancen und Schäden von Maßnahmen, wie zum Beispiel die Beteiligung an einem Reallabor, sorgfältig ab. Nach Auffassung von Kepplinger (2009) gehen Akteure der Politik, wenn diese Erfolgsaussichten sehen, relativ große Risiken ein (auch im Vergleich zu anderen Akteuren). So zeigt eine Befragung von Kepplinger (2009), dass die meisten befragten Politiker(innen) der Ansicht sind, dass 
Politik irrational handeln würde, wenn Politiker(innen) das Risiko von Fehlschlägen scheuten. Dieser Widerspruch zwischen vergleichsweise hohen Kosten im Fall von Kritik und der Selbsteinschätzung als risikoaffine Menschen erklärt sich möglicherweise aus der Frage, wie gut entstandene Schäden auf einzelne Handelnde zurückgeführt und Konsequenzen einzelnen Personen zugeordnet werden können. Alles in allem ist das Teilsystem Politik risikoaffin. Dies zeigte sich beispielsweise auch in den BaWü-Labs, die vielfach von der Kommunalpolitik bereits in der Anfangsphase unterstützt wurden, obwohl aufgrund der zahlreichen Unsicherheiten ein nicht geringes Risiko bestand.

Gegenstand des Reallabors Wissensdialog Nordschwarzwald (,BaWü-Lab WiNo“) war der Nationalpark Schwarzwald, der erste Nationalpark in Baden-Württemberg. Obwohl dessen Einrichtung politisch umstritten war, unterstützten einzelne Bürgermeister(innen) der Region das Reallabor ausgesprochen engagiert, z. B. durch die Mitwirkung bei öffentlichen Veranstaltungen. Dies kann zum einen damit begründet werden, dass die kommunalen Vertreter(innen) davon ausgingen, dass ein Scheitern dieser Veranstaltungen bzw. des gesamten Projekts nicht ihnen persönlich angelastet werden würde. Zum anderen betraf ihr Engagement nicht originäre Verwaltungs- bzw. hoheitliche Aufgaben ihrer Kommunen, sondern lag im Bereich freiwilliger Leistungen. So stehen Gemeinden beispielsweise im Tourismus auch in Konkurrenz zueinander und streben die Herausarbeitung von Alleinstellungsmerkmalen an. Ein Teilprojekt des Reallabors wiederum befasste sich damit, nachhaltige Tourismusprodukte auf Basis des Waldes zu entwickeln. Bürgermeister(innen), die sich engagierten, nahmen daher auch eine unternehmerische Haltung ein, die zu höherer Risikoaffinität führte.

Risikomanagement: Das Teilsystem Politik kann aufgrund seiner Risikoaffinität auf ein breites Spektrum von Risikomanagementstrategien zurückgreifen. Dies umfasst vorsorgende Strategien wie den Verantwortungstransfer auf andere Akteure durch „Schwarze Peter-Spiele“ bis hin zu Strategien zum Umgang mit Schäden wie z. B. das „Framing von negativen Meldungen“. In den BaWü-Labs konnte als eine Risikomanagementstrategie insbesondere beobachtet werden, dass die Politik die Kontrolle darüber bewahrte, in welchem Umfang sie öffentlich mit dem Projekt assoziiert wurde.

Im Rahmen des Reallabors Stadt:Quartiere 4.0 - Frühzeitige gestaltende Bürgerbeteiligung für eine nachhaltige Entwicklung in Baden-Württemberg (,,BaWü-Lab Stadt: Quartiere 4.0") entstand im Zuge der Arbeit eine Differenz zwischen den Forschungsergebnissen und den politischen Zielen der beteiligten politischen Akteure, so dass sich deren anfängliche Risikoaffinität über den Projektzeitraum hin zu größerer Zurückhaltung bis hin zur Risikoaversion veränderte. Die Risikomanagementstrategie der politischen Akteure bestand darin, darauf hinzuwirken, dass Resultate aus der wissenschaftlichen Arbeit nur insoweit kommuniziert werden sollten, als sie politischen Zielen zuträglich schienen, und die Veröffentlichung von Ergebnissen, die diesen abträglich schienen, zumindest nicht zu unterstützen. Dies wiederum schränkte den Handlungsspielraum des Reallabors insofern ein, als einige der Arbeiten im Reallabor eine umfängliche Kommunikation der Ergebnisse an die breite Öffentlichkeit vorgesehen hatten. 
Im BaWü-Lab BUGA:log beteiligte sich die Stadt Heilbronn, vertreten durch die Person des Oberbürgermeisters und den Gemeinderat, sowohl über eine erhebliche finanzielle Unterstützung am Reallabor als auch durch die aktive Mitarbeit an der Konzeption und Umsetzung des Reallabors. Durch die intensive Mitarbeit in der Konzeptionsund Vorbereitungsphase betrieb die Stadt Heilbronn für sich selbst auch aktives Risikomanagement, indem sie ihre Positionen und Bedürfnisse (z. B. im Bereich des Verkehrsrechts, aber auch im Bereich der Lade- und Versorgungsinfrastruktur) früh einbrachte, so dass von Beginn an Risiken mit den Partnern aus der Wissenschaft diskutiert und Lösungen dafür entwickelt werden konnten.

\subsection{Recht}

Recht - Der Begriff des Rechts bezieht sich vor allem auf Einrichtungen, die der Rechtsprechung und Rechtspflege zuzuordnen sind, also beispielsweise Gerichte oder auch Genehmigungsbehörden, die vor allem die Einhaltung geltenden Rechts gewährleisten sollen. Entsprechend institutionell angebundene, individuelle Gesellschaftsmitglieder, deren Aufgabe in der Rechtsprechung und Rechtspflege liegt, werden ebenfalls dem Teilsystem Recht zugeordnet.

Funktion, Leitwert und Leitdifferenz: Die Funktion des Teilsystems Recht ist es, normative Erwartungen zu stabilisieren, indem es für die Gesellschaft verlässliche Verhaltensregeln entwickelt, festhält und Sanktionen für deren Nichteinhaltung festlegt (Callies 2006). Leitwert ist die Feststellung von Recht mit der Leitdifferenz ,Recht/Unrecht'. Rechtmäßig ist eine Handlung, wenn sie geltenden Rechtsvorgaben des in einer Gesellschaft dem Teilsystem Recht zugrundeliegenden Gesetzeskörpers entspricht. Aufgrund dieses über alle gesellschaftlichen Teilsysteme hinweg geltenden Gesetzeskörpers spielt das Teilsystem Recht für alle anderen Teilsysteme eine begrenzende, aber auch eine ermöglichende Rolle. Kein Teilsystem kann außerhalb des vom Teilsystem Recht festgelegten Rahmens also außerhalb des Gesetzes - agieren, selbst wenn sein jeweils eigener Leitwert und seine eigenen Leitdifferenzen dies durchaus zulassen würden. Umgekehrt ermöglicht das Teilsystem Recht jedoch auch über die Teilsysteme hinweg verlässliche Handlungsabläufe durch eine klare, regulatorische und normenbasierte Rahmung. Das würde sich bei einer nicht rechtsgeleiteten Auseinandersetzung allein auf der Grundlage der verschiedenen Handlungslogiken der anderen Teilsysteme nicht ergeben.

Qualitätssicherung: Qualitätssicherung erfolgt im Teilsystem Recht über den Abgleich eines Sachverhalts mit geltenden Gesetzen, die Rechtsaufsicht durch Gerichte sowie die Sanktionierung im Fall einer Nichteinhaltung von Recht.

Entscheidungsstrukturen: Die Prüfung, ob ein Sachverhalt geltendem Recht entspricht, erfolgt oft mehrstufig, von einer ersten laienhaften Einschätzung bis hin zu einer richterlichen Entscheidung. Die Entscheidung, welche Informationen im Sinne des Leitwerts, Recht/Unrecht' anschlussfähig an das Teilsystem sind, un- 
terliegt im Teilsystem Recht einer starken Professionalisierung. Die Professionalisierung besteht in einer notwendigen juristischen (Teil-)Ausbildung und dem stark formalisierten Nachweis juristischen Könnens. Es gibt jedoch auch Positionen, wie beispielsweise das deutsche Verfassungsgericht, in das Richter(innen) durch die Politik berufen werden, womit es hier eine „Inter-Systembeziehung“ zum Teilsystem Politik gibt.

Finanzierung: Die Finanzierung von Rechtsstrukturen wie Ämtern und Gerichten erfolgt in erster Linie aus Steuermitteln, während der individuelle Zugang zum Rechtssystem auch aus privaten Mitteln gewährleistet wird. Dabei wird im Teilsystem Recht stark darauf geachtet, dass der Zugang grundsätzlich allen Bürger(inne)n offensteht: Um diesen für jedermann zu gewährleisten, werden, in Abhängigkeit von Vorleistungen und individuellen Vermögenslagen, kollektiv gestützte Prozesskostenhilfen über Versicherungen sowie über Transferzahlungen angeboten.

Schäden und Chancen: Das Teilsystem Recht ist in transdisziplinären (und transformativen) Forschungsprojekten insbesondere durch die jeweiligen (z. B. kommunalen) Verwaltungen repräsentiert. Der Extremfall eines Schadens aus Sicht des Teilsystems Recht ist, wenn im Rahmen von beispielsweise Realexperimenten rechtlich nicht zulässige Maßnahmen umgesetzt werden. Dies kollidiert klar mit der Leitdifferenz ,Recht/Unrecht' und widerspricht dem Selbstverständnis der kommunalen Verwaltung, Regeln einzuhalten und geltendem Recht zu entsprechen (Hill 2016). Schäden aus Sicht der Verwaltung ergeben sich zudem aus Haftungsrisiken in derartigen Projekten. Risiken entstehen, wenn beispielsweise kommunale Verwaltungen innovative Maßnahmen für die Umsetzung von Realexperimenten genehmigen müssen, eventuell für diese Entscheidung in die Haftung genommen werden und somit die Leitdifferenz von ,Recht/Unrecht` zum Tragen kommt. Dies kann in einem Reallabor beispielsweise zur Folge haben, dass selbst wenn die fachlich Zuständigen in einer kommunalen Verwaltung willens wären, eine innovative Maßnahme zu unterstützen, diese durch die hausinternen Jurist(inn)en mit Verweis auf die Amtshaftung gebremst werden. Transdisziplinäre (und transformative) Forschungsprojekte bieten kommunalen Verwaltungen aber auch die Chance, Maßnahmen auszuprobieren, die im üblichen Verwaltungshandeln nicht möglich wären. Dies, indem Reallabore einen Transfer von Risiken von der kommunalen Verwaltung auf die Wissenschaft, die die rechtliche Verantwortung übernimmt und ggf. haftet, ermöglichen.

In Karlsruhe ergab sich im Laufe des BaWü-Labs GO Karlsruhe die rechtliche Möglichkeit, neuartige Fahrbahnmarkierungen zur Fußgängerförderung auszuprobieren. Die Kommune bat daher das BaWü-Lab, diese neuen Möglichkeiten in Form eines Realexperiments umzusetzen und deren Wirkung wissenschaftlich zu analysieren, bevor diese generell in der Stadt umgesetzt würden. Neben der Möglichkeit der schnellen Umsetzung der Maßnahmen spielte hierfür auch der teilweise Risikotransfer auf die Akteure der Wissenschaft eine Rolle. 
Beim Realexperiment „Parklets für Stuttgart“ (s. auch Köglberger et al. 2019 in diesem Buch, Kapitel 4) des BaWü-Labs RNM wurden straßenbegleitende Parkplätze zu Aufenthaltsflächen für Fußgänger(innen) umgestaltet. Hierdurch entstand zum einen das Unfallrisiko eines Zusammenstoßes zwischen Fahrzeugen und einem Parklet. Zum anderen hätten beim Aufbau oder bei der Nutzung der Parklets Fußgänger(innen) auf die Straße treten können, so dass es auf diese Weise zu Unfällen mit Personenschäden hätte kommen können. Für diese möglichen Schäden hätte die Universität als Selbstversicherer gehaftet. Um diese Risiken zu minimieren, wurden im Rahmen der Genehmigung durch das Amt für öffentliche Ordnung Auflagen erlassen, für deren Einhaltung die Wissenschaftler(innen) Sorge tragen mussten. So wurde die Verkehrssicherheit für vorbeifahrende PKW durch das Anbringen von reflektierenden Folien erhöht, beim Aufbau der Parklets wurde die Baustelle sichtbar markiert und abgesperrt, und die Aufbauenden trugen Warnwesten. Die Parklets durften nur vom Bürger(innen)steig aus zugänglich sein, um ein Hinaustreten auf die Straße zu verhindern. Da keine Partei über Erfahrungen mit einer vergleichbaren Intervention hatte, wurde die Risikobewertung teilweise auch nachträglich noch angepasst, woraus kurzfristige Nachbesserungsforderungen entstanden.

Risikoaversion und Risikoaffinität: Im Vergleich zu den anderen Akteuren in transdisziplinären (und transformativen) Forschungsprojekten verhalten sich Verwaltungen eher risikoavers. Hill (2016) und Fischer (2016) zeigen auf, dass ein vorsichtiger Umgang mit Risiken das Verhalten von Verwaltungen generell kennzeichnet. Die Folge ist ein auf Abstimmung und Absicherung bedachter Umgang mit Risiken. Risiken werden nur akzeptiert, wenn diese erforderlich sind, um das Allgemeinwohl zu gewährleisten. Nach dieser Handlungslogik ist ein Risiko nur dann akzeptabel, wenn institutionelle Regeln bestehen, um diese zu kontrollieren (Renn 2008).

Im BaWü-Lab GO Karlsruhe sollten im Rahmen eines Realexperiments temporäre Bauteile zur Geschwindigkeitsreduktion verbaut werden. Für diese Bauteile wurde von der kommunalen Verwaltung entsprechend geltendem Recht gefordert, dass sie einschlägigen Prüfsiegeln entsprechen. Jedoch ergab eine umfassende Recherche, dass für Bauteile dieser Art in der Planungspraxis keine Prüfsiegel oder Qualitätsnormen bestehen. Der Anforderung des Rückgriffs auf etablierte Regelwerke konnte daher nicht entsprochen werden. Daraufhin erfolgte durch die Verwaltung eine ausführliche Abfrage bei anderen Kommunen, welche Erfahrungen bisher mit solchen Bauteilen gemacht worden waren und welche institutionellen Regeln zur Anwendung gekommen waren. Erst gestützt darauf erfolgte die Entscheidung der Verwaltung, das Realexperiment zu ermöglichen.

Risikomanagement: Als generelle Risikomanagementstrategien von Verwaltungen nennt Fischer (2016) das ausgeprägte Festhalten an Regeln und Hierarchien. Daher wird in einer Verwaltung jedes von den Regeln abweichende Verhalten tendenziell als bedrohlich aufgefasst. Die Arbeit in transdisziplinären (und transformativen) Forschungsprojekten, insbesondere die Umsetzung innovativer Maßnahmen im Rahmen z. B. von Realexperimenten, hat jedoch häufig zur Folge, dass das Festhalten an etablierten institutionellen Regeln als Risikomanagement- 
strategie nicht möglich ist. Bei der Mitarbeit in derartigen Projekten muss die kommunale Verwaltung daher gewillt sein, sich über ihre etablierten Risikomanagementstrategien hinwegzusetzen. Umgekehrt müssen die Akteure aus der Wissenschaft gewillt sein, sich den Risikomanagementstrategien der Verwaltung durch institutionelle Regeln anzupassen, soweit es solche gibt.

Im BaWü-Lab BUGA:log in Heilbronn zeigte sich, dass komplexen rechtlichen Anforderungen, die sich aus dem autonomen Fahren im öffentlichen Straßenraum ergaben, am besten mit einem in zeitlicher Hinsicht parallel zum Projekt stattfindenden Dialogprozess begegnet werden konnte. In diesen Dialog wurden früh alle in rechtlicher Hinsicht relevanten Entscheidungsebenen (,,von der Kommune bis zum Bund“) einbezogen. Früh wurde auch das Ziel formuliert, eine gemeinsame Lösung erarbeiten zu wollen, also nicht rechtliche Verantwortlichkeiten zwischen Ebenen hin und her zu schieben. Als Leitprinzip diente dabei der Wunsch, den Interessen des Reallabors so weit wie möglich entsprechen zu können, aber gleichzeitig auch ohne Einschränkungen die Möglichkeiten und Grenzen aller beteiligten Verwaltungsebenen bei der Einhaltung und Durchsetzung des geltenden Rechtsrahmens zu respektieren.

Im Rahmen des BaWü-Labs Stadt:Quartiere 4.0 waren die Wissenschaftler(innen) des Projekts zwar in die Konzeption von Veranstaltungen im Rahmen des Integrierten Mobilitätsentwicklungsplans (IMEP) der Stadt Herrenberg eingebunden, jedoch gab es klare Grenzen in Bezug auf die Visionen nachhaltiger Mobilität, die dabei entwickelt werden konnten. So konnten gewisse Visionen, die durch die Simulationen und Visualisierungen des Reallabors hätten generiert werden können, nicht weiterverfolgt werden, weil sie nicht im Interesse der Kommune lagen. Das Risiko für die Kommune wurde durch die kommunalen Akteure dadurch reduziert, dass eine Konzentration auf die konkrete Umsetzung von Maßnahmen stattfand und die Kontrolle des Prozesses sowie der Öffentlichkeitsbeteiligung soweit als möglich bei der Verwaltung und dem Gemeinderat gelassen wurden.

\subsection{Wirtschaft}

Wirtschaft - Unter dem Begriff der Wirtschaft werden Einrichtungen und individuelle Gesellschaftsmitglieder verstanden, deren Ziel es ist, über Marktmechanismen ihren individuellen oder gruppenbezogenen Nutzen in Form von Profit zu steigern. Dazu gehören primär Unternehmen.

Funktion, Leitwert und Leitdifferenz: Als Funktion der Wirtschaft gelten die Allokation $^{6}$ von Gütern beziehungsweise, die „Kommunikation von Knappheit [...] im Medium des Markts“ beziehungsweise die Verknüpfung zukunftsfähiger Vorsorge mit je gegenwärtigen Bedürfnissen (Luhmann 1988, S. 63). Dabei befreit sie die Produzent(inn)en vom unwahrscheinlichen Finden der Nachfrage nach ihren Produkten, indem sie Angebot und Nachfrage zusammenkommen lässt.

6 Allokation ist die Zuweisung von finanziellen Mitteln, Materialien und Produktivkräften. 
Gleichzeitig ermöglicht sie eine vergleichsweise konfliktfreie Vornahme von Tauschhandlungen, da der Erwerb von Gütern über die Zahlung von Geld erfolgt, das nur in Form einer Weitergabe nutzbar ist (Luhmann 1984). Unterschiedliche Gesellschaften fokussieren auf unterschiedliche Knappheiten und wählen unterschiedliche Ansätze, um „diese Knappheit durchzusetzen“, beispielsweise über einen Plan oder über den Markt (Baecker 2016, S. 169). Die handlungslogische Rahmung des Teilsystems mit dem Leitwert der Erhaltung und des Zuwachses der Zahlungsfähigkeit eines Unternehmens oder eines individuellen Gesellschaftsmitglieds sowie der Leitdifferenz ,Zahlung/keine Zahlung' ist breit diskutiert. Preise erlauben dabei eine Kommunikation über zu erwartende Zahlungen und eine Reduktion des Tauschvorgangs auf Zahlungen, ohne anderweitige Verpflichtungen entstehen zu lassen. Preise sind also als „Informationsraffung und -verkürzung“ (Luhmann 1988, S. 19) zu verstehen. Innerhalb der Handlungslogik des Teilsystems Wirtschaft ist eine Handlung nur anschlussfähig, wenn ein Austausch weitere Zahlungen ermöglicht, also Gewinn verspricht. Jedoch agieren nicht alle Akteure aus der Wirtschaft, die einem Unternehmen zuzuordnen sind, ausschließlich nach einer wirtschaftlichen Handlungslogik. Vielmehr wird diese oft ergänzt durch die Logik der Corporate Social Responsibility, die eine am Gemeinwohl orientierte Verantwortung als ergänzenden Leitwert postuliert und damit das Kriterium ,gesellschaftlich wertvoll'. So haben Unternehmen die Wahl, ob sie sich aufgrund von Gewinnerwartungen oder, ähnlich wie zivilgesellschaftliche Akteure (s. Abschnitt 3.5), aufgrund der gesellschaftlichen Verantwortung an einer Unternehmung beteiligen.

Qualitätssicherung: Die Entstehung von Profit ist als Qualitäts- und Erfolgsindikator für das Teilsystem Wirtschaft zu betrachten, da sie den Handlungsspielraum des Akteurs, der den Profit empfängt, im Hinblick auf zukünftige Zahlungen erweitert.

Entscheidungsstrukturen: Hierarchien und Entscheidungsstrukturen erklären sich aus der Zahlungsfähigkeit, der Liquidität, die einem Akteur im Teilsystem Wirtschaft gegenwärtig oder ggf. auch zukünftig zugeschrieben wird. Dies kann direkt der Fall sein, indem auf das eigene Vermögen des Akteurs abgestellt wird, die Zuschreibung kann auch indirekt erfolgen, indem auf die Zuschreibung der Befähigung zur Zahlungsfähigkeit durch Banken abgestellt wird.

Finanzierung: Wirtschaft ist das einzige Teilsystem, in dem Entscheidungen über Finanzierungen nicht als Produkt der Umwelt zu verstehen sind, sondern im Teilsystem selbst entstehen. Das Medium Geld stellt den Mittelpunkt von Kommunikationsabläufen dar. Das erklärt, warum der vielbemühte ,Business Case ${ }^{7}$ von

7 „Ein Business Case ist ein Szenario zur betriebswirtschaftlichen Beurteilung einer Investition. Auch ein Projekt stellt eine Investition dar und muss deshalb seine Vorteilhaftigkeit gegenüber der Geschäftsleitung unter Beweis stellen“ (Brugger 2009, S. 11). 
solcher Relevanz ist: Aus Teilsystemperspektive muss eine Zahlung eine weitere Zahlung ermöglichen, um das selbstreferenzielle System aufrechtzuerhalten.

Schäden und Chancen: Die Akteure aus dem Teilsystem Wirtschaft beurteilen die mit Schäden und Chancen verbundenen Risiken von transdisziplinären (und transformativen) Forschungsprojekten vorrangig nach der Leitdifferenz ,Zahlung/keine Zahlung' bzw. dem daraus abgeleiteten Profit. Dabei sind die Akteure der Wirtschaft bereit, das Risiko von Schäden in Form von monetären Verlusten in Kauf zu nehmen, wenn die Chance besteht, (ggf. später) Gewinne zu erzielen.

Im Reallabor Schorndorf: Zukunftsweisender Öffentlicher Verkehr - Bürgerorientierte Optimierung der Leistungsfähigkeit, Effizienz und Attraktivität im Nahverkehr (,,BaWü-Lab Schorndorf") (s. auch Steckbrief im Anhang zu diesem Buch) waren der Verkehrs- und Tarifverbund Stuttgart (VVS), der lokale Busbetreiber sowie die Kommune als Projektpartner in das Projekt eingebunden. Alle Beteiligten sahen in der Erprobung eines neuen, bedarfsgerechten Bussystems (ohne festen Fahrplan und fixe Haltestellen) die Chance herauszufinden, ob ein solches Bussystem die Zukunft des ÖPNV sein könnte. Um dieses zukünftige Potenzial ausloten zu können, brachten die Praxispartner eigene Ressourcen dafür ein, die zunächst einmal eine ,Mehrbelastung ‘ darstellten. Diese wurden beispielsweise dazu eingesetzt, im Rahmen des Testbetriebs im Reallabor den Kund(inn)en ein vergünstigtes Busticket anzubieten.

Im BaWü-Lab Energielabor Tübingen mit der Zielrichtung „Nachhaltige Energiewende" wurde u. a. mit einem lokalen Energieversorger kooperiert. Dieser musste, wie alle anderen Energieversorger auch, mit der laufenden Transformation des Energiesystems umgehen. Diese soll, nach unverändert geltender Auffassung, im Sinne der Nachhaltigkeit vor allem dezentral ablaufen, was aktuelle Geschäftsmodelle in Frage stellt, neue rechtliche Fragestellungen aufwirft und die technische Komplexität steigen lässt. Demgegenüber verfügte der Energieversorger nur über ein begrenztes Budget für Investitionen in Forschung und Entwicklung. Solange aber noch niemand weiß, wo genau die Reise hingeht, sind alle Beteiligten zurückhaltend, wenn es darum geht, in eine bestimmte Richtung - hier in Richtung neuer dezentraler Energieversorgungssysteme - zu gehen, so auch der Praxispartner im Reallabor. Das wirkte sich auch auf die Arbeit im Reallabor aus. Zum einen schränkte es den Handlungsspielraum ein, zum anderen waren die betriebswirtschaftlichen Erwägungen seitens des Energieversorgers zu berücksichtigen.

Im Reallabor Urban Office - Nachhaltige Stadtentwicklung in der Wissensgesellschaft (,,BaWü-Lab Urban Office“) (s. auch Steckbrief im Anhang zu diesem Buch) wurde mit einem Unternehmen der Stadt Heidelberg zusammengearbeitet. Es war zunächst schwierig, das Unternehmen für die Kooperation zu gewinnen, da der Geschäftsführer keinen direkten Profit damit verbinden konnte. Für ihn stellte sich das Projekt zunächst ausschließlich als Risiko dar, da Ressourcen investiert werden mussten, ohne einen möglichen Gewinn abschätzen zu können. Erst im Projektverlauf gelang es, nach einigen Gesprächen und ersten Arbeitsschritten, dem Geschäftsführer die Chancen des gemeinsamen Projekts aufzuzeigen und ihn so von den potenziellen Chancen einer $\mathrm{Zu}$ sammenarbeit zu überzeugen. 
Risikoaversion und Risikoaffinität: Akteure aus der Wirtschaft weisen generell eine gewisse Risikoaffinität auf. Sie folgen dabei einer entrepreneurhaften Logik und sind bereit, Risiken im Austausch für entstehende Chancen zu akzeptieren (Renn 2008; Hill 2016). Diese generelle Risikoaffinität von Akteuren aus der Wirtschaft konnte auch in den BaWü-Labs beobachtet werden. Für transdisziplinäre (und transformative) Forschungsprojekte bedeutet dies, dass wirtschaftliche Akteure bereit sind, Ressourcen wie Zeit und Geld in die Arbeit an einem derartigen Projekt zu investieren. Dabei nehmen sie das Risiko in Kauf, dass die Investitionen verloren gehen können, solange die Chance besteht, von den Ergebnissen des Projekts zu profitieren.

Im BaWü-Lab Space Sharing wurden, als ein Teilaspekt, rechtliche Fragestellungen bearbeitet. Um das tun zu können, wurde ein Praxispartner aus der Wirtschaft mit Fachexpertise im Immobilienrecht ins Projektteam integriert. Dieser war insofern bereit, ein Risiko einzugehen, als er Zeit und auch Geld in Form von Mitarbeiter(innen)stunden investierte, ohne damit direkten Gewinn zu erzielen. Er sah aber die Chance, aus den gewonnenen Erkenntnissen zukünftig wirtschaftlichen Nutzen ziehen zu können, indem er diese im ziemlich jungen Markt von Immobilien für Mehrfachund Parallelnutzung - mit entsprechend geringen Erfahrungswerten - würde anwenden können.

Im BaWü-Lab BUGA:log engagierten sich mehrere Industriepartner größtenteils unentgeltlich mit der Bereitstellung von Komponenten für das Fahrzeug und die Reallabor-Umgebung. Sogar erheblicher Umbau- und Anpassungsaufwand an eigenen Komponenten wurde teilweise in Kauf genommen, weil die möglichen Chancen, die sich aus einem Erfolg des Reallabors ergeben könnten, als sehr hoch bewertet wurden.

Risikomanagement: Akteure aus der Wirtschaft nutzen für ihr Risikomanagement häufig Ansätze aus dem mathematischen Risikoverständnis. Das monetäre Risiko wird dabei als Produkt aus Schadenshöhe und Eintrittswahrscheinlichkeit betrachtet. Im Ergebnis können die Akteure für ihr Risikomanagement eine klare Grenze ableiten, welches finanzielle Risiko sie für einen erwarteten Gewinn durch ein transdisziplinäres (und transformatives) Forschungsprojekt akzeptieren. Ein Potenzial derartiger Projekte ist, dass die Akteure der Wirtschaft die Möglichkeit haben, einen Teil des Risikos auf andere Akteure (z. B. aus der Wissenschaft) zu verlagern. Die Risiken von Schäden werden so aus der jeweils eigenen Gewinnund Verlustrechnung herausgehalten und anderen Akteuren überlassen (Kromschröder 2003). In vielen transdisziplinären (und transformativen) Forschungsprojekten übernehmen die Akteure der Wissenschaft das höhere finanzielle Risiko, da sich diese am Ziel Wissensgewinnung und nicht am Ziel Profit orientieren. 


\subsection{Zivilgesellschaft}

Zivilgesellschaft - Unter dem Begriff Zivilgesellschaft werden Einrichtungen und individuelle Gesellschaftsmitglieder verstanden, deren Handeln sich nicht primär aus einem rechtlichen oder politischen Auftrag oder aus den Zielen Wissensgewinnung oder Profit erklärt, sondern vor allem aus dem Ziel, sich individuell oder als Gruppe an gemeinwohlorientierten, also nicht an Partikularinteressen orientierten, gesellschaftlichen Gestaltungsprozessen zu beteiligen.

Funktion, Leitwert und Leitdifferenz: Ob die Zivilgesellschaft ein Teilsystem im Sinne Luhmanns und Schimanks ist, ob sich also eine durchgängige Handlungslogik herausarbeiten lässt, kann nicht eindeutig beantwortet werden. Dies hat sicher zum einen mit der teilweise überaus breiten Definition von Zivilgesellschaft zu tun (Reichel 2012, S. 56), die mitunter den gesamten gesellschaftlichen Raum zwischen Staat und Wirtschaft erfasst, und zum anderen mit der Betonung der ,Verteidigungsrolle' und damit Abwehrhaltung der Zivilgesellschaft gegenüber Staat und Wirtschaft. So umfasst der Begriff häufig sowohl den Sportverein als auch die Bürgerrechtsbewegung. Dies verdeutlicht intuitiv, weshalb die Frage, ob tatsächlich ein kohärentes gesellschaftliches Teilsystem vorliegt, noch nicht abschließend diskutiert ist. Wer davon ausgeht, dass die Zivilgesellschaft ein gesellschaftliches Teilsystem ist, setzt keinen gemeinsamen, dem gesamten Teilsystem zugrunde liegende Wertekonsens nach Reichel voraus. Das heißt, es ist nicht relevant, ob sich die Werte der einzelnen Akteure unterscheiden oder sogar widersprechen, wichtig ist nur, dass sie Werte als die wesentliche Grundlage ihres Handelns mit dem Ziel gesellschaftlicher Gestaltung erachten. Als Leitwert wird also die gemeinnützige, das heißt über den persönlichen Nutzen hinausgehende und auf der Grundlage von Werten erfolgende Gestaltung gesellschaftlicher Bedingungen gesehen.

Qualitätssicherung: Eine Handlung ist demnach im Sinne des Teilsystems Zivilgesellschaft qualitativ gut und erfolgreich, wenn sie Werte umsetzt oder gesellschaftliche Anschlüsse an diese verstärkt oder erschließt. Dies wird erreicht, indem Menschen wertebezogen davon überzeugt werden, sich einer Gruppe oder Organisation von wertemäßig Gleichgesinnten anzuschließen oder indem sich Gleichgesinnte zu neuen wertebasierten Gruppen oder Organisationen zusammenfinden. In jedem Fall besteht der unbedingte Anspruch der Akzeptanz der entsprechenden grundliegenden Werte durch die Mitglieder der Gruppe bzw. der Organisation.

Entscheidungsstrukturen: Hierarchien in zivilgesellschaftlichen Zusammenhängen leiten sich häufig daraus ab, wie authentisch wertebezogen jemand lebt bzw. wie authentisch jemand für die Werte der Gruppe oder Organisation wirbt. Durch Gleichgesinnte bekommen Akteure, innerhalb geltenden Rechts, einen größeren Handlungs- und Entscheidungsspielraum zugesprochen. 
Finanzierung: Die Zivilgesellschaft übernimmt in geringerem Maße festgelegte und definierte staatliche Aufgaben - außer in Trägerschaft - und verfügt dadurch meist über eine nur geringfügige langfristige, institutionelle Förderung. Ein häufiges Finanzierungsmodell sind Spenden durch Gleichgesinnte als Ausdruck einer wertemäßigen Übereinstimmung der spendenden Akteure mit dem jeweiligen Organisationszweck oder eher kürzere Förderungen in Form von Fördermitteln, die beispielsweise von staatlichen Einrichtungen kommen können oder von anderen gemeinnützigen Organisationen. Dies gilt insbesondere für kleine Initiativen und Bewegungen ohne langfristige Aufgabe und gesellschaftlich zugeschriebene Rolle und kann dazu führen, dass langfristige Planungen in den Hintergrund treten.

Schäden und Chancen: Schäden können sich ergeben, wenn durch Maßnahmen im Rahmen eines transdisziplinären (und transformativen) Forschungsprojekts die Werte des Akteurs aus der Zivilgesellschaft angestrebt, diese dann aber in der Umsetzung verfehlt werden. Der Schaden für die Akteure der Zivilgesellschaft besteht darin, dass die von der Teilnahme an einem solchen Projekt erhoffte Unterstützung der eigenen Werte nicht erfolgt. Wenn die angestrebten Ergebnisse verfehlt werden, verlieren die betroffenen Akteure die Legitimation für die eingesetzten Ressourcen. Ein weiterer Schaden kann entstehen, wenn in einem transdisziplinären (und transformativen) Forschungsprojekt den Werten der Akteure widersprechende Ergebnisse erzielt werden. Zivilgesellschaftliche Akteure müssen sich dann insbesondere innerhalb ihrer jeweiligen Organisationen rechtfertigen, warum sie an einem solchen Projekt mitgewirkt und das Ergebnis nicht verhindert haben. Die Ergebnisoffenheit zivilgesellschaftlicher Akteure ist also vergleichsweise geringer als beispielsweise die der Akteure aus der Wissenschaft. Die Chancen für zivilgesellschaftliche Akteure sind, dass durch das transdisziplinäre (und transformative) Forschungsprojekt die Akzeptanz und Verbreitung der eigenen Werte gefördert werden. Zudem besteht in solchen Projekten die Chance, Gleichgesinnte für die zu vertretenden Werte zu finden. Insbesondere transdisziplinäre (und transformative) Forschungsprojekte, deren Themenstellungen aus einer Werteperspektive anschlussfähig sind, können solche Chancen für die Zivilgesellschaft eröffnen.

In Heidelberg eröffnete die Zusammenarbeit mit Wissenschaftler(inne)n den zivilgesellschaftlichen Akteuren im BaWü-Lab Urban Office neue Chancen für die Thematisierung von alternativen Perspektiven auf aktuelle Stadtentwicklungsprozesse. Unterschiedliche Akteure wie Architekt(inn)en, Städteplaner(innen), Student(inn)en und Stadtforscher(innen) fanden sich zusammen und gründeten den Verein „Urban Innovation - Stadt neu denken! e. V.“ mit Sitz in Heidelberg. Der Verein konnte ein Urban Innovation Center in einem zur Zwischennutzung freigegebenen Gebäude eröffnen und stellte dort, über die Laufzeit des Reallabors hinaus, Co-Working-Arbeitsplätze zur Verfügung, mit dem Ziel, Bürgerschaft, Wirtschaft, Wissenschaft und Kommunalpolitik zusammenzubringen, um gemeinsam innovative Lösungen für Herausforderungen in Heidelberg zu entwickeln. 
Im Reallabor 131: KIT findet Stadt (,BaWü-Lab R131“) (s. auch Steckbrief im Anhang zu diesem Buch) zeigten sich die Chancen und Risiken für zivilgesellschaftliche Akteure auf unterschiedlichen Projektebenen. Zivilgesellschaftliche Akteure ergriffen in Form von Realexperimenten (,Dein NachhaltigkeitsExperiment“, vgl. Trenks et al. 2018) die Chance, die Umsetzung eigener Werte und Zielvorstellungen in der Praxis zu testen. Im Zentrum standen dabei Themen wie Gemeinschaftsbildung, Schutz der Biodiversität oder Entschleunigung. Des Weiteren brachten sie sich als Praxisakteure in transdisziplinäre und transformative Bildungsprozesse ein und nutzten die Möglichkeit, gemeinsam mit Studierenden die eigene Arbeit voranzutreiben und ihre Sichtbarkeit zu stärken (s. Beecroft 2019 in diesem Buch). Für zivilgesellschaftliche Akteure stellten die eingebrachten personellen Ressourcen den Engpass dar, der mit Blick auf die Chancen und Risiken einer gemeinsamen Aktivität entscheidend war, da die Wirksamkeit der einzelnen Aktivitäten und deren positive öffentliche Resonanz hoch sein konnten, aber nicht garantiert waren. Ein weiteres Risiko konnte sich aus einer zu hohen Intensität der im Reallabor umgesetzten Interventionen ergeben, da diese durchaus zu Ergebnissen führen konnten, die dann im normalen Arbeitsmodus der zivilgesellschaftlichen Akteure kaum aufrechtzuhalten waren.

Risikoaversion, Risikoaffinität: Aus der Handlungslogik des Teilsystems Zivilgesellschaft ergibt sich keine klare Präferenz für die Bereitschaft zum Risiko. Beispielsweise waren die in BaWü-Labs eingebundenen zivilgesellschaftlichen Akteure überaus divers, was deren Risikobereitschaft anbelangt. Das deutet darauf hin, dass die Risikobereitschaft zivilgesellschaftlicher Akteure stärker als in den anderen Teilsystemen von den involvierten Personen abhängt und weniger von der Handlungslogik des Teilsystems. Die zivilgesellschaftlichen Gruppen und Personen tendieren dazu, jeweils individuell abzuwägen, ob die sich durch ein Projekt ergebenden Chancen das mit einer Beteiligung verbundene Risiko rechtfertigen.

Risikomanagement: Das Gleiche trifft auf die Frage des Risikomanagements zu. Ein über alle Akteure der Zivilgesellschaft hinweg ähnliches Risikomanagement ist nicht erkennbar.

\section{Risiken, Schäden und Chancen durch das Zusammentreffen teilsystemspezifischer Handlungslogiken}

Wie in den vorangegangenen Kapiteln dargestellt wurde, wirken unterschiedliche teilsystembezogene Handlungslogiken und Risikokulturen in transdisziplinäre (und transformative) Forschungsprojekte hinein. Dabei muss beachtet werden, dass die Handlungen, Schwerpunkte und Positionen einzelner Personen nicht ausschließlich von ihrer Teilsystemzugehörigkeit und von den Leitwerten des jeweiligen Teilsystems abgeleitet werden können, sondern auch auf persönlichen Vorlieben, eigenen Erfahrungen und anderen individuellen Eigenschaften beruhen. Gleichwohl werden die Akteure, um kommunikativ an ihr Teilsystem anschluss- 
fähig bleiben zu können, die jeweilige Handlungslogik im Blick haben und sich auch im Sinne der jeweils spezifischen Risikokultur verhalten. Im Folgenden wird zunächst beleuchtet, was geschieht, wenn unterschiedliche Handlungslogiken aufeinandertreffen. Im Anschluss wird diskutiert, welche Chancen und Risiken sich daraus ergeben können und welche Rolle Risikoaffinität und -aversion in diesem Zusammenhang spielen.

\subsection{Was geschieht, wenn die Handlungslogiken unterschiedlicher Teilsysteme aufeinandertreffen?}

Wenn die Handlungslogiken unterschiedlicher Teilsysteme aufeinandertreffen, bedeutet dies zunächst, dass die gleiche in einem Projekt geplante Aktivität unterschiedliche Bewertungen erfährt hinsichtlich der Passung zu einem Leitwert, der Anschlussfähigkeit an die bisherige Arbeit, der notwendigen Maßnahmen zur Qualitätssicherung, der Art und des Ausmaßes bestehender Risiken und/oder des möglichen Risikomanagements. Das folgende Beispiel beleuchtet das Zusammentreffen unterschiedlicher Handlungslogiken hinsichtlich der Anschlussfähigkeit einer Befragung an unterschiedliche Teilsysteme, die über die jeweilige Leitdifferenz ersichtlich wird.

Im Reallabor „Asylsuchende in der Rhein-Neckar-Region“ (,,BaWü-Lab Asylsuchen$d e^{\text {") }}$ (s. auch Steckbrief im Anhang zu diesem Buch) wurden Daten zur Einstellung von Anwohner(inne)n in Bezug auf die Einrichtung von Unterkünften für Geflüchtete durch die Wissenschaft als besonders anschlussfähig erachtet. Untersuchungen dazu hatten zum damaligen Zeitpunkt ein besonders hohes Erkenntnispotenzial, da die dezentrale Unterbringung von Geflüchteten in verschiedenen Stadtteilen einen neuen, noch wenig erforschten Steuerungsansatz darstellten. Es war davon auszugehen, dass die Peer-Diskussion und wissenschaftlichen Begutachtungen das Potenzial derartiger Ergebnisse als vielversprechend einstufen würden. Entsprechend groß war das Interesse der beteiligten Wissenschaftler(innen) an der Datengewinnung. Da zum geplanten Befragungszeitpunkt jedoch in der betreffenden Kommune die Standortfrage von Unterkünften noch nicht entschieden war, musste auf kommunaler Seite zunächst geklärt werden, welches Signal eine derartige Befragung in die Stadt- oder Ortsteildiskussion geben könnte. Hier bestand die Sorge, dass Fragen zu Einstellungen gegenüber einer potenziellen Unterbringung von Geflüchteten in den entsprechenden Stadtteilen als Ankündigung einer tatsächlichen Unterbringung gewertet werden würden und die Stadtverwaltung deswegen mit Gegenwehr aus den befragten Stadtteilen zu rechnen hätte. Die Befürchtung war, dass ein schwieriger Dialogeinstieg mit der Bürgerschaft eine möglicherweise tatsächlich irgendwann einzurichtende Unterbringung verhindern würde, weil das Thema, verbrannt` wäre. Gesellschaftliche Gestaltung basierend auf kollektiven Entscheidungen wäre dann unmöglich, weil die durch die Befragung vorab ausgelöste Diskussion mit den Bürger(inne)n den politischen Gestaltungsspielraum verringert hätte. Das Vorgehen wurde also aus Sicht des Teilsystems Politik als wenig anschlussfähig erachtet. 
An diesem Beispiel lässt sich ein durch das Zusammentreffen zweier unterschiedlicher Handlungslogiken entstehender Widerspruch sehr schön erkennen: Die wissenschaftliche Anschlussfähigkeit einer Erkenntnis wird durch ihre Zitierung in relevanten Journals belegt. Eine Erkenntnis ist dabei insbesondere dann anschlussfähig, wenn sie im Teilsystem Wissenschaft so zum ersten Mal dargestellt wird, also wenn beispielsweise Daten präsentiert und analysiert werden, die in dieser Form noch nicht vorliegen. Im Teilsystem Politik wiederum ist die Übersetzbarkeit einer Maßnahme in politische Unterstützung ein Qualitätsmerkmal. Droht durch die Durchführung einer Maßnahme vor allem der Entzug politischer Unterstützung in der Wählerschaft, so wird sie entsprechend der Leitdifferenz von ,Macht/keine Macht' nicht als qualitativ wertvoll eingestuft.

Fragen der multiperspektivischen Qualität einer Maßnahme können also sehr unmittelbar den Weg in die Diskussion leiten, ob ein Akteur in einem transdisziplinären (und transformativen) Forschungsprojekt eine Maßnahme unterstützt und wenn ja, in welchem Umfang. Dabei kann die Beurteilung aus der Perspektive von mehr als nur zwei Teilsystemen erfolgen. Hierzu ein Beispiel, bei dem Akteure aus Wissenschaft, Politik und Zivilgesellschaft an der Diskussion einer Maßnahme beteiligt waren, bei der es um die Erweiterung des Forschungsgegenstands ging.

Im Reallabor STADT-RAUM-BILDUNG - Reallabor für die nachhaltige Planung von Bildungslandschaften und die Integration von Aus- und Umbauten von Schulgebäuden (,,BaWü-Lab SRB“) (s. auch Steckbrief im Anhang zu diesem Buch) wurden gemeinsam mit Schulträgern (kommunalen Verwaltungen) und mehreren Schulen anhand von Simulationsexperimenten mögliche zukünftige, vor allem bauliche Veränderungen (Transformationen) der Schulen und des Quartiers erarbeitet. Um ein fruchtbares Resultat mit Blick auf die Nutzbarkeit der Forschungsergebnisse zu erzielen, war es wichtig, auch eine zunächst strukturelle Erweiterung der Schule für die Oberstufe und den Abschluss der allgemeinen Hochschulreife mitzudenken, die sich auch baulich auswirken würde. Eine solche Erweiterung wird jedoch auf politischer Ebene und in der Öffentlichkeit sehr kontrovers diskutiert, da solche Schulen meist als Konkurrenz zu Gymnasien empfunden werden. Einige Elternvertreter(innen) als Akteure aus der Zivilgesellschaft wie auch Akteure aus der Verwaltung und aus der Wissenschaft begrüßten die Erweiterungsoption. Die Eltern setzten sich dafür ein, weil die dann möglichen, neuen Bildungsschwerpunkte den von ihnen unterstützten Werten in der Schulbildung entsprachen, die kommunale Verwaltung, weil sich die planerische Sicherheit im Umgang mit steigenden Schülerzahlen erhöhen würde, und die Wissenschaft, weil sie Erkenntnisse aus den neuen Bildungssettings gewinnen könnte. In allen drei Teilsystemen erwies sich die Erweiterungsoption also als grundsätzlich anschlussfähig. Die tatsächliche Entscheidung über eine Erweiterung lag jedoch beim demokratisch gewählten Gremium, dem Gemeinderat, also bei einem weiteren Akteur aus dem Teilsystem Politik. Hier wurde ein Abrücken von bisherigen Bildungsschwerpunkten nicht unbedingt als eine Maßnahme erachtet, die größte Wählerunterstützung erfahren würde, womit sich die Anschlussfähigkeit ans Teilsystem Politik letztlich als schwieriger erwies. Um diesen möglichen Konflikt zwischen Vertreter(inne)n der genannten 
Teilsysteme von vornherein abzumildern, wurde eine Erweiterung für die Oberstufe als zusätzliche Option gestaltet, so dass die Transformation in zwei Stufen - mit und ohne Erweiterung - diskutiert werden konnte.

Schwierig an der Teilsystemvielfalt ist bei diesem Beispiel unter anderem die Einschätzung, welcher Akteur welchem Teilsystem zuzuordnen ist und ob diese Zuordnung durchgängig durch die Debatte eingehalten wird. So können beispielsweise die Elternvertreter(innen) sowohl als Akteure der Zivilgesellschaft als auch als Akteure im politischen System handeln, indem sie sich entweder wertebezogen für bestimmte Bildungsschwerpunkte einsetzen oder sich als potenzielle Wähler(innen) verhalten. Realitätsnah dürfte in solchen Fällen, insbesondere an der ,Grenze' zwischen Zivilgesellschaft und Politik, die Annahme sein, dass es eine Mischung der beiden Handlungslogiken ist, die die Wahrnehmung, die Bewertung und das Handeln der Beteiligten steuert.

Ein weiteres Beispiel für unterschiedliche Perspektiven auf und Bewertungen desselben Sachverhalts in Abhängigkeit des Teilsystems ist der hinlänglich diskutierte Umgang mit dem Faktor Zeit. Während beispielsweise im Teilsystem Politik verstreichende Zeit bis zur Entwicklung einer Lösung für ein gesellschaftliches Problem in einem Machtverlust resultieren kann, ist ein so direkter Zusammenhang im Teilsystem Wissenschaft nicht herzustellen, d. h. der Faktor Zeit ist der wissenschaftlichen Qualität untergeordnet.

So beteiligten sich Akteure aus der Kommune, und damit aus den Teilsystemen Recht und Politik, aufgrund eines akuten Lösungsbedarfs für gesellschaftspolitische Fragen am BaWü-Lab Asylsuchende. Hier war es die Notwendigkeit der Integration von Geflüchteten nach 2015. Dieser Lösungsbedarf war im Sinne des Teilsystems Politik akut, da unzufriedene Wähler(innen) zu Machtverlust führen können. Auch im Teilsystem Recht spielte der Faktor Zeit eine wichtige Rolle, da im Gesetz verankerte, einzuhaltende Fristen die Grenze zwischen Recht und Unrecht markieren können. Für die Wissenschaft aber, die auf theoretischen Fragestellungen aufbaute, war dieser Faktor von untergeordneter Bedeutung. Wichtiger war den Akteuren aus diesem Teilsystem, dass Aussagen zu Effekten und ,Good Practices', die als Lösungen in Frage kommen, möglicherweise erst nach Abschluss des gesamten Forschungsprozesses, also nach drei Jahren getroffen werden können. Grund für diese Vorsicht war der Zeitbedarf, der üblicherweise nötig ist für die intensive Beobachtung beispielsweise mehrerer Fälle sowie für eine wissenschaftlichen Ansprüchen genügende Generierung und Auswertung von Daten.

Eine Herausforderung kann auch der Umgang mit rechtlichen Risiken bieten. So ist für die meisten Vertreter(innen) aus der Wissenschaft die Berücksichtigung von rechtlichen Risiken eine ungewohnte Aufgabe. Dies im Gegensatz zu beispielsweise kommunalen Verwaltungen. Erschwerend kommt oft hinzu, dass sich Realexperimente in rechtlich und verfahrenstechnisch nicht durchdeklinierten Handlungsräumen abspielen, da sie aufgrund ihres experimentellen Charakters außerhalb standardisierter Genehmigungs- und Gestaltungsprozesse abgewickelt werden müssen. 
Im BaWü-Lab Space Sharing mussten sich die Akteure aus der Wissenschaft als Betreiber des Realexperiment-Standorts Space Sharing mit rechtlichen Anforderungen der Liegenschaftseigentümer sowie der Nutzer(innen) auseinandersetzen. Das bedeutete, dass es für sie auf einmal die Leitwertdifferenz ,Recht/Unrecht' zu berücksichtigen galt, die im Teilsystem Wissenschaft nur am Rande eine Rolle spielt. Gleichzeitig gab es für das Modell der parallelen und zeitversetzten Nutzung derselben Räumlichkeiten durch unterschiedliche Personen und für verschiedene Nutzungen noch kein vorgefertigtes rechtliches Vertragsrahmenwerk oder entsprechende Erfahrungswerte. Deswegen wurde dieser Aspekte gezielt als eigene Fragestellung herausgearbeitet und als Teil des Forschungsprojekts durch einen Praxispartner mit entsprechender Fachexpertise bearbeitet.

Nicht immer muss das Aufeinandertreffen von Handlungslogiken zu einem Konflikt führen, auch ein bloßes Feststellen eines Spannungsfelds bzw. einer Überlagerung kann mit Blick auf einen konstruktiven Umgang damit schon hilfreich sein.

\subsection{Welche Chancen und Grenzen ergeben sich aus dem Zusammentreffen unterschiedlicher Handlungslogiken?}

In allererster Annäherung ergibt sich aus dem Zusammentreffen unterschiedlicher Handlungslogiken einerseits die Chance, dass sich der Handlungsspielraum eines Projekts und somit sein Erfolgspotenzial vergrößern, und andererseits das Risiko, dass sich der Handlungsspielraum und somit sein Erfolgspotenzial verringern. Beides soll im Folgenden illustriert werden.

Das Zusammentreffen zweier oder mehrerer Teilsysteme und ihrer Handlungslogiken kann zu einer Chance werden, wenn beispielsweise eine Handlung in den Bereich des Möglichen rückt, die in der Handlungslogik nur eines der beteiligten Teilsysteme zwar wünschenswert, aber nicht möglich wäre. Dies kann der Fall sein, wenn z. B. für ein Vorhaben ein bestimmtes, neues Wissen notwendig ist, dessen Produktion sich jedoch nicht mit dem Leitwert des entsprechenden Teilsystems vereinbaren lässt und deswegen keine Mittel dafür bereitgestellt werden können. Vergleichsweise häufig tritt dieser Fall bei Realexperimenten auf, die beispielsweise für eine kommunale Verwaltung oder einen zivilgesellschaftlichen Akteur durchgeführt und wissenschaftlich begleitet werden.

Im BaWü-Lab Asylsuchende wurden in einer Kooperation zwischen einer Hochschule und einem großen Bildungsträger sogenannte Lerntheken entwickelt. Dabei handelt es sich um teildigitalisierte Lernstationen, an denen - unterstützt von einer Fachlehrkraft und/oder ehrenamtlichen Sprachhelfer(inne)n - erste Deutschlernschritte unternommen werden können. Diese Lerntheken wurden insbesondere für die Lebenssituation in Erstaufnahmezentren entwickelt, in denen sich Deutschunterricht im Klassenverband aufgrund der Terminstreuung der Geflüchteten, des kurzen und versetzten Verbleibs in der Aufnahmestelle und gegebenenfalls fehlender Mittel nicht umsetzen lässt. Der 
Bildungsträger verfügte weder über die personellen Möglichkeiten noch über die wissenschaftliche Expertise, die Lerntheken ohne Unterstützung aus dem Teilsystem Wissenschaft zu entwickeln. Eine auf Forschung ausgerichtete wissenschaftliche Arbeit mit dem Ziel des Erkenntnisgewinns, wie solche Lerntheken aufzusetzen sind, entsprach nicht seinen bildungsbezogenen Kernanliegen. Umgekehrt fehlten der wissenschaftlichen Seite für die Reflexion der Lernsituation Erstaufnahmestelle und für ihre pädagogische Begleitung die Perspektive auf die praktischen Notwendigkeiten, das Wissen um die Rahmenbedingungen und generell die Einbindung in das Aufnahmesystem von Geflüchteten. Die tatsächliche Schaffung außeruniversitärer Bildungssettings entspricht nicht dem Leitwert des Teilsystems Wissenschaft. Erst durch die Kooperation rückte für beide Akteure die Entwicklung der Lerntheken in den Bereich des Möglichen.

Im BaWü-Lab Energielabor Tübingen trafen zwei Handlungslogiken aufeinander. Die eine folgte der Devise ,Alle Ressourcen in die praktische Umsetzung konkreter Energiewende-Maßnahmen stecken" und wurde insbesondere von einem zivilgesellschaftlichen Praxispartner eingebracht, der seit Langem daran arbeitete. Die andere folgte der Devise „Es gilt, die Ursachen der aktuellen Nichtumsetzung besser zu verstehen“ und wurde von den Forscher(inne)n stark gemacht. Zur Vermittlung zwischen diesen Logiken erwies es sich als sehr hilfreich, die politischen Gegebenheiten (Reform des Erneuerbare-Energien-Gesetzes, keine Internalisierung externer Kosten bei Treibhausgasen etc.), an denen das Reallabor nicht ansetzen konnte, als problematische Rahmenbedingung der Arbeit ausdrücklich auszuweisen und zu akzeptieren. Dies wirkte handlungsentlastend, weil sich damit das Reallabor diesen Fragen nicht spezifisch widmen musste, sie also aus der Arbeit ausklammern konnte. Zugleich wurden aber im Bereich der Energiesuffizienz, der dem Praxispartner am Herzen lag, konkrete Experimente zum Thema Klimaschutz und Gutes Leben (Wendepunktprojekte) durchgeführt, die die Dimension des direkten und indirekten Energiebedarfs und -konsums adressierten. Die wissenschaftliche Begleitung und Auswertung dieser Wendepunktprojekte ermöglichten eine Adressierung beider Handlungslogiken.

Der Handlungsspielraum kann sich insbesondere dann verringern, wenn die potenziellen Schäden aus der Perspektive mehrerer gesellschaftlicher Teilsysteme ins Blickfeld geraten. So können insbesondere Risikowahrnehmungen aus dem Teilsystem Recht relevant werden.

Das Realexperiment „Stäffele Gallery“ des BaWü-Labs RNM beschäftigte sich mit der Frage, wie die zahlreichen Treppen, die bei der bewegten Stuttgarter Topografie wichtige Wegverbindungen für Fußgänger(innen) darstellen, verbessert werden können. Dafür wurden verschiedene Interventionen geplant und durchgeführt, die sich nicht nur auf die Treppen selbst, sondern auch auf die ihnen angegliederten oder von ihnen gekreuzten Straßenräume bezogen. Um das Risiko von Unfällen mit Personenschäden zu vermeiden, untersagte die genehmigende Behörde jedoch verschiedene der angedachten Maßnahmen, wie beispielsweise Markierungen, die Zebrastreifen ähnelten, da diese bei Fußgänger(inne)n ein trügerisches Sicherheitsgefühl hervorrufen könnten. Hier war es klar die Risikowahrnehmung aus dem Teilsystem Recht, die zu einer Einschränkung des Handlungsspielraums führte. 
Aufgrund der großen Ausdifferenzierung und Regulierungsdichte im Teilsystem Recht besteht häufig nur sehr wenig Spielraum für Kosten-Nutzen-Abwägungen bei innovativen oder von der Norm abweichenden Prozessen. Für Akteure, die dem Teilsystem nicht angehören, ist oft nicht zu erkennen, wie sie in einer innovativen, also nicht oder wenig regulierten Situation rechtlich abgesichert handeln können. Die Zuschaltung eines Akteurs aus dem Teilsystem Recht kann jedoch aufgrund des Professionalisierungsgrads in diesem Teilsystem die Kosten für den Prozess deutlich erhöhen. Das wiederum kann dazu führen, dass eine Intervention, deren Durchführung in einem rein wissenschaftlichen, experimentellen Setting unproblematisch wäre, in der ,realen Welt' nicht durchführbar ist, und zwar auch dann nicht, wenn die Durchführung des Realexperiments geltendem Recht - beispielsweise der Straßenverkehrsordnung - nicht explizit widerspricht. Aufgrund des engen Regelsystems des Teilsystems Recht ist es bereits ausreichend für die Unzulässigkeit des Realexperiments, wenn eine experimentelle Situation rechtlich nicht geregelt ist.

Weitere Begrenzungen des Handlungsspielraums eines Projekts können durch unterschiedliche rechtliche Sanktionsformen beispielsweise im Zusammenhang mit Haftungsregelungen entstehen. Mit unterschiedlichen Risikobewertungen verbunden ist hier die Frage der Haftung. Im Teilsystem Wissenschaft wird die Haftung im Normalfall nicht von einzelnen Wissenschaftler(inne)n, sondern von deren Institution getragen. ${ }^{8}$ Für Schäden, die durch die Verwendung oder Bekanntgabe von Forschungsergebnissen entstehen, besteht eine weitreichende Haftungsfreiheit für lege artis gewonnene Erkenntnisse mit einer Verpflichtung zur sofortigen Berichtigung anerkannter Irrtümer und unter Ausschluss des Tatbestands leichter Fahrlässigkeit (Heldrich 2015). Aufgrund der bei Forschung in der ,realen Welt' potenziell höheren Eintrittswahrscheinlichkeit sowie höheren Schadenshöhe von Ereignissen hingegen kann es erforderlich sein, dass Akteure der Wissenschaft vorsichtiger agieren, dass der Handlungsspielraum also verringert wird, um Risiken auszuschließen, die zwar nicht aus der Sicht des Teilsystems Wissenschaft ein Risiko darstellen, sehr wohl aber aus der Sicht des Teilsystems Recht.

Im BaWü-Lab GO Karlsruhe wurden verschiedene Realexperimente im öffentlichen Verkehrsraum durchgeführt. Damit bestand das Risiko, dass durch die Realexperimente Verkehrsunfälle mit Sach- oder Personenschäden entstehen. Daher wurde von den Akteuren aus der Wissenschaft besonders sorgfältig darauf geachtet, sicherzustellen, dass durch die Realexperimente keine über die übliche Unfallgefahr hinausgehenden

8 Jedoch kann eine Schadensersatzpflicht für individuelle Versuchsleiter(innen) entstehen, wenn vorab nicht ausreichend über die Risiken eines (Real-)Experiments informiert wurde oder wenn es zu Verletzungen der Sorgfaltspflicht kam. 
Gefährdungen entstehen. Als an einem Standort während der Versuchsphase ein Verkehrsunfall passierte, war es wichtig, dass aus der Unfallbeschreibung nachvollzogen werden konnte, dass dieser nicht ursächlich auf das Realexperiment zurückzuführen war. Andernfalls wäre die Fortsetzung der Realexperimente bedroht gewesen.

\subsection{Was bedeuten Risikoaversion und Risikoaffinität beim Zusammentreffen unterschiedlicher Handlungslogiken?}

Wenn sich Akteure in der Handlungslogik unterschiedlicher gesellschaftlicher Teilsysteme bewegen, richten sie ihr Augenmerk auf jeweils unterschiedliche Risiken. Durch die abweichende Risikoidentifikation können Verständnisschwierigkeiten entstehen, wenn beispielsweise zwar die aus einem anderen Teilsystem stammende Risikoeinschätzung zur Kenntnis genommen, aber die damit einhergehende Risikoaversion zum Vorwurf gemacht wird.

Im BaWü-Lab GO Karlsruhe fasste ein Teil der zivilgesellschaftlichen Akteure das von rechtlichen Risiken geleitete Verhalten der Kommune als Verweigerungshaltung auf, frei nach dem Motto, dass die Stadt sich nicht traue, den geäußerten Bürgerwillen umzusetzen, sich also nicht - wie die Vertreter(innen) der Zivilgesellschaft - wertorientiert verhalte. Nicht bei allen Akteuren aus der Zivilgesellschaft konnte ein Verständnis dafür geweckt werden, dass das regelkonforme Risikoverhalten der Kommune der auf sie einwirkenden Handlungslogik des Teilsystems Recht entsprach und daher zu respektieren war.

Aus dem Zusammenwirken unterschiedlicher, den Handlungslogiken der jeweiligen Teilsysteme folgenden Risikokulturen können sich Chancen ergeben. So kann beispielsweise ein in der risikoaversen Handlungslogik seines Teilsystems handelnder Akteur seinen Handlungsspielraum indirekt, durch die Kooperation mit einem in der risikoaffinen Handlungslogik eines anderen Teilsystems handelnden Akteur erweitern. Dies ist häufig für kommunale Verwaltungen als risikoaversem Akteur der Fall, wenn sie mit Vertreter(inne)n der Wissenschaft, die in einer risikoaffinen Handlungslogik agieren, kooperieren. So kann es innovative Infrastrukturmaßnahmen geben, die als ,Regelmaßnahmen“ für eine Kommune nicht möglich sind, weil es für sie (noch) keine allgemeine Regelung gibt. Werden sie jedoch wissenschaftlich begleitet und damit als ,Experiment" gekennzeichnet, so können sie über das experimentelle Setting und die dem Teilsystem Wissenschaft zugeordnete Verantwortung im Testlauf durchgeführt werden. In einem nächsten Schritt können dann positive Ergebnisse aus solchen experimentellen Settings als Argument für eine rechtliche Anpassung und allgemeine Regelung solcher innovativer Infrastrukturmaßnahmen dienen. Dadurch wird es den Kommunen möglich, nach einem Testlauf derartige Maßnahmen als Normalfall umzusetzen. Hier stellen sich also eine Risikobetrachtung, die das Gesamtprojekt in den Blick nimmt, und die dann daraus resultierende Zuordnung des Risikos auf den jeweils risikoaffineren Akteur als Chance der Kooperation dar. 
On-Demand Mobilitätsangeboten, wie dem Bedarfsbus im BaWü-Lab Schorndorf, wird ein hohes Potenzial mit Blick auf die Gestaltung der Mobilität von morgen zugesprochen. Die rechtlichen und regulatorischen Randbedingungen erlauben allerdings die flexible Bedienform nicht ohne Weiteres. Für Experimente jedoch erlaubt das Personenbeförderungsgesetz (PBefG) eine praktische Erprobung für einen beschränkten Zeitraum von maximal vier Jahren (gestützt auf die sogenannte „Experimentierklausel“, § 2 Abs. 7 PBefG). Durch die Zusammenarbeit im Reallabor wurde es möglich, das Potenzial einer innovativen Betriebsweise zu erproben. Ob eine Umsetzung als Normalfall möglich wäre, müsste geprüft werden (gestützt auf die „Auffangklausel“", $\S 2$ Abs. 6 PBefG).

Wird umgekehrt die Risikobewertung durch die Akteure jeweils einzeln vorgenommen, ist für die Entscheidung meist das geringste akzeptierte Risiko der jeweils vertretenen Teilsysteme ausschlaggebend. Ein Realexperiment kann beispielsweise nicht realisiert werden, wenn das Risiko schon nur eines Akteurs überschritten wird. Der ,kleinste gemeinsame Risikonenner ${ }^{6}$ wird dann selbst zum Risiko für das Projekt. Aufgrund des unterschiedlichen Verständnisses von Risiko kann diese Entscheidung zudem für die anderen Akteure nur schwer nachvollziehbar sein, was zu Spannungen im Projekt führen kann.

Im BaWü-Lab GO Karlsruhe hatten die wissenschaftlichen und die kommunalen Akteure teilweise unterschiedliche Einschätzungen zum akzeptablen Risiko von Realexperimenten zu Maßnahmen zur Fußgängerförderung im öffentlichen Straßenraum. Beide Akteure waren sich einig, dass der Verkehrssicherheit ein hohes Gewicht einzuräumen ist. Uneinigkeit bestand hingegen darüber, welche Folgewirkungen der Maßnahmen für andere Verkehrsteilnehmer(innen) (Umwege, Parkraum etc.) akzeptabel seien. Das Risiko von Folgewirkungen wurde entsprechend der den beteiligten Teilsystemen innewohnenden Risikoaffinität und -aversion unterschiedlich eingeschätzt. Da die Kommune die verkehrsrechtlichen Anordnungen erlässt, war deren Risikobewertung letztendlich ausschlaggebend.

\section{Welches Risikomanagement ist in Anbetracht unterschiedlicher Handlungslogiken und Risikokulturen zu empfehlen?}

In Kapitel 3 dieses Beitrags wurden die Handlungslogiken ausgewählter in transdisziplinären (und transformativen) Forschungsprojekten wie Reallaboren vertretenen gesellschaftlichen Teilsysteme anhand zentraler Charakteristika herausgearbeitet. Zudem wurden Risikobewertungen, Risiko und übliche Risikomanagementansätze in diesen Teilsystemen dargestellt. Auf dieser Grundlage wurde in Kapitel 4 anhand von Beispielen gezeigt, wie sich das Zusammentreffen der unterschiedlichen Handlungslogiken und Risikokulturen in Projekten auswirken kann. Im Folgenden werden nun drei Empfehlungen gegeben, wie sich aufbauend auf den Erfahrungen der BaWü-Labs das Zusammentreffen der unterschiedlichen 
Handlungslogiken und Risikokulturen in transdisziplinären (und transformativen) Forschungsprojekten konstruktiv begleiten lässt. Diese sind ein aktives Risikomanagement (Abschnitt 5.1), die Analyse und Anpassung der Risikoverteilung (Abschnitt 5.2) sowie das bewusste Antizipieren von Risiken (Abschnitt 5.3).

\subsection{Aktives Risikomanagement betreiben}

Die Literatur (vgl. u. a. Motel und Richter 2016; Link 2001; Hill 2016) empfiehlt für das Zusammenspiel von Akteuren mit unterschiedlichen Handlungslogiken die Anwendung eines aktiven Risikomanagements. Diese Empfehlung kann auch auf transdisziplinäre (und transformative) Forschungsprojekte übertragen werden, und sie lässt sich auch aus den Erfahrungen der BaWü-Labs ableiten. Ein Risikomanagement erweitert den Handlungsspielraum für die Kooperation der verschiedenen Akteure, da Risiken früher identifiziert werden können und daher mehr Zeit zur Verfügung steht, mit diesen Risiken proaktiv umzugehen (Motel und Richter 2016). Ein umfassendes Risikomanagement mit den Schritten Risikoidentifikation, Risikobewertung und Risikosteuerung wurde in keinem der BaWü-Labs aktiv realisiert, in einzelnen BaWü-Labs wurden jedoch einzelne Schritte eines Risikomanagements durchgeführt, die zeigen, dass ein solches bewusstes Risikomanagement möglich ist.

In einer sehr frühen Projektphase des BaWü-Labs BUGA:log fand ein erster Workshop mit allen beteiligten Akteuren statt, in dem es darum ging, eine gemeinsame Projektzeitplanung aufzusetzen sowie kritische Pfade und Abhängigkeiten zwischen einzelnen Arbeitspaketen zu benennen. Dieser Plan enthielt also auch Elemente der Risikoidentifikation. Er diente im weiteren Projektverlauf allen Akteuren des Reallabors als Orientierung und gab gleichzeitig eine Struktur für die regelmäßigen Projektsitzungen vor. Diese Form der Strukturierung wurde von allen Projektpartnern akzeptiert und für erforderlich gehalten, insbesondere auch, weil der Zeitraum, in dem das Realexperiment durchgeführt werden sollte - die Bundesgartenschau 2019 -, von Beginn an klar und fest vorgegeben war. Da damit ein Verschieben des Realexperiments unmöglich war und da gleichzeitig die Nichtumsetzung des Realexperiments Konsequenzen für die Bundesgartenschau gehabt hätte, war es von zentraler Bedeutung, die Unwägbarkeiten sehr bewusst in die Projektplanung aufzunehmen.

Zum Ersten zeigt die Erfahrung der BaWü-Labs bezüglich der Fragen des Risikomanagements, dass es wichtig ist, nicht nur auf Risiken im Sinne von Schäden $\mathrm{zu}$ fokussieren, sondern auch Chancen sprachlich und analytisch durchgehend im Blick zu haben. Eine solche Chance ist beispielsweise eine mögliche Erweiterung des Handlungsspielraums der Akteure im Zusammenspiel der Handlungslogiken und Risikokulturen (s. die Beispiele in den Abschnitten 4.2 und 4.3). Die systematische Analyse von Chancen und Risiken aus der Perspektive der jeweils wirkenden Handlungslogiken und Risikokulturen erlaubt es, diese Chancen und Risiken nicht aus dem Blick zu verlieren und damit die Handlungssicherheit aller 
Beteiligten und des Projekts insgesamt zu erhöhen. Die verschiedenen Akteure haben aufgrund der für sie relevanten Handlungslogiken oft auch unterschiedliche Kompetenzen entwickelt. Werden diese Kompetenzen im gesamten Projektverlauf im Blick behalten, ist es möglich, jeweils den Akteur mit einer Aufgabe zu betrauen, für den diese nicht neu ist, weil sie in besonderer Weise seiner Handlungslogik entspricht. Wenn beispielsweise ein Reallabor dazu beitragen will, bestimmte Veränderungen im Verhalten einer Stadtbevölkerung im Sinne einer nachhaltigeren Lebensführung zu bewirken, gilt es, die Kompetenzen der Akteure aus den Teilsystemen Politik und Zivilgesellschaft bezüglich der Gestaltung der Gesellschaft zu nutzen. Das Teilsystem Politik kann hierbei z. B. sowohl auf Mechanismen der Macht als auch der Vorbildwirkung zurückgreifen, während das Teilsystem Zivilgesellschaft z. B. über Beispiele, Vernetzung und die Ansprache von Werten Einfluss nehmen kann.

Zum Zweiten sind in den unterschiedlichen Handlungslogiken Erfolg und Misserfolg nicht gleichartig definiert. Erfolg und Misserfolg sind eine nachträgliche Bewertung, die sich an der ursprünglichen Zielsetzung des jeweiligen Akteurs bemisst (siehe Kapitel 2). Es empfiehlt sich, ein besonderes Augenmerk auf die unterschiedlichen, aus den verschiedenen Handlungslogiken resultierenden Auffassungen von Schaden zu legen - insbesondere mit Blick auf die Langzeitfolgen, die diese mit sich bringen können. Hier sind beispielsweise Schäden aus Sicht des Teilsystems Recht zu nennen, da diese oft Haftungsfragen beinhalten und somit im Eintrittsfall des Schadens schwerwiegende Auswirkungen auf die Träger des Risikos, auch über den Projektzeitraum hinaus, haben können. Klare Absprachen sowie Bewusstmachung dieser rechtlichen Risiken bei allen Beteiligten sind notwendig. Gegebenenfalls kann es sinnvoll sein, parallel zu oder vor Realexperimenten das entsprechende rechtliche Setting auszuarbeiten. Ein weiteres relevantes Beispiel sind politische Schäden, die gebührend beachtet werden sollten. Ein Realexperiment, das zum Beispiel die Bevölkerung zu stark polarisiert, kann die Umsetzung eines politischen Projekts auf Jahre hinaus unmöglich machen, weswegen ein detailliertes Risikomanagement hier von Vorteil wäre.

Zum Dritten erlaubt es eine nach Handlungslogiken differenzierte Analyse von Erfolg und Misserfolg, die verengte Erfolgsbewertung im Sinne von ,erfolgreich/ gescheitert" für das Gesamtprojekt zu überwinden. So kann beispielsweise ein vorab entwickeltes, nach Handlungslogiken ausdifferenziertes Chancen- und Risikenraster dazu beitragen, über das gesamte Projekt hinweg einen detaillierten Überblick über Erfolgs- und Misserfolgsaspekte des Projekts zu haben. Ein solches Raster ist für die Steuerung wie für die interne und externe Kommunikation eines Projekts dienlich.

Konkrete Maßnahmen des Risikomanagements lassen sich leicht verständlich für alle Beteiligten umsetzen (ohne die Notwendigkeit, vorher ein komplexes Risikomanagement-Regelwerk verstehen zu müssen). Dies ist bei Bedarf auch kurzfristig 
und mit beschränktem Zeitbudget möglich. Aufgrund der für transdisziplinäre (und transformative) Forschungsprojekte wichtigen Vorteile empfiehlt es sich daher, solche unabhängig von Größe oder zeitlichem Umfang des Forschungsprojekts durchzuführen. Je größer und komplexer das Projekt wird, umso sinnvoller kann es sein, ein bewusstes Risikomanagement über das Gesamtprojekt aufzubauen. Teil eines umfassenden Risikomanagements eines transdisziplinären (und transformativen) Forschungsprojekts sollte sein, dass zu Beginn eine Reflexion über die im Projekt vorhandenen Handlungslogiken und Risikokulturen stattfindet und dass Maßnahmen ergriffen werden, die das Verständnis zwischen den Vertreter(inne)n der verschiedenen Teilsysteme für die jeweils anderen Handlungslogiken und Risikokulturen erhöhen.

Bei der Planung und Umsetzung des Risikomanagements sowie einzelner Maßnahmen sollten auf jeden Fall der zeitliche sowie der personelle Aufwand berücksichtigt werden sowie Überlegungen zum Verhältnis von Aufwand und Nutzen angestellt werden. Maßnahmen zum Aufbau von Verständnis für die unterschiedlichen Handlungslogiken und die damit einhergehenden Risikokulturen sind kurzfristig aufwendig, können aber zu einem späteren Zeitpunkt im Projekt Konflikte und zeitliche oder finanzielle Verluste ersparen. Auch längerfristige Zerwürfnisse zwischen Projektbeteiligten können auf diese Weise verhindert werden. Ein komplexes Regelwerk zum Risikomanagement oder zeitaufwendige Verpflichtungen, wie die Teilnahme an umfangreichen Workshops zur gemeinsamen Risikoidentifikation und zur Definition von Maßnahmen zur Risikostreuung, können aber potenzielle Akteure auch von einer Beteiligung abschrecken.

\subsection{Risikoverteilung analysieren und bei Bedarf anpassen}

In Reallaboren sollten, wie generell in Innovationskooperationen, die Chancen und Risiken fair auf die Akteure aufgeteilt werden. Während beim traditionellen Risikomanagement die getrennt vorgenommenen Einzelrisikobewertungen der verschiedenen Akteure zum Risiko des Projekts insgesamt zusammengefügt werden, bietet sich nach Link (2001) bei Innovationskooperationen ein umgekehrtes Vorgehen an. Dabei wird beispielsweise in einem Reallabor zuerst das Risiko eines Realexperiments aus übergeordneter Sicht betrachtet. In einem nächsten Schritt wird dann analysiert, wie dieses Risiko auf die einzelnen Akteure im Reallabor aufgeteilt wird. Als Ausgangslage sind in transdisziplinären (und transformativen) Forschungsprojekten Risiken oft insofern ungleich verteilt, als das, was aus Sicht eines Teilsystems als Risiko zu werten ist, von Akteuren aus einem anderen Teilsystem verursacht wird.

In Reallaboren besteht zum Beispiel, abhängig vom Forschungsgegenstand, häufig eine ungleiche Risikoverteilung zwischen Forscher(inn)en und kommunalen Akteuren, da die Forscher(innen) Risiken verursachen, die von der Verwaltung 
mitgetragen werden müssen. Dabei kommt noch hinzu, dass der Nutzen, der aus einer Inkaufnahme des Risikos entsteht, in der Handlungslogik des einen Teilsystems relevant ist, der Schaden jedoch in der eines anderen. Insbesondere bei Projekten, die technische Strukturen aufbauen, ist der Frage besondere Aufmerksamkeit zu widmen, ob Nutzen und Schaden jeweils einseitig in einer bestimmten Handlungslogik zu verorten sind. Bei Realexperimenten, die im Stadtraum erfolgen, können auch Externe und eventuell dem Forschungsgegenstand gegenüber Ablehnende von den Auswirkungen betroffen sein. Dies kann besonders dann zu Problemen führen, wenn Wissenschaftler(innen) ein Realexperiment mit dem Ziel wissenschaftlichen Erkenntnisgewinns durchführen, in der Öffentlichkeit aber die Kommune als Eignerin der technischen Struktur und somit als Verantwortliche wahrgenommen wird. Hier entsteht eine Diskrepanz zwischen jenen, die einen Gewinn aus dem Realexperiment ziehen (bspw. in Form von Daten für Publikationen), und jenen, die Risiken (bspw. Bürgerbeschwerden oder sogar Klagen) tragen.

Im BaWü-Lab GO Karlsruhe wurden Maßnahmen zur Verbesserung der Situation des Fußverkehrs gemeinsam mit zu Fuß Gehenden entwickelt und provisorisch umgesetzt und im Sinne einer wissenschaftlichen Handlungslogik getestet. Um die Chance der Wissensgenerierung und der Förderung des Fußverkehrs zu haben, waren Eingriffe in den öffentlichen Verkehrsraum erforderlich. Als Maßnahme zur Temporeduzierung in Wohnstraßen kam der Wunsch auf, im Rahmen eines Realexperiments die Wirksamkeit von Fahrbahnschwellen zu testen. Diese werden deutschlandweit eingesetzt, verfügen aber nicht über eine explizite Zulassung, da es für Fahrbahnschwellen und Kissen keine eindeutigen Anforderungen in Form von technischen Lieferbedingungen oder Ausführungsbestimmungen gibt. Der Einbau muss daher von der jeweiligen Straßenverkehrsbehörde genehmigt werden, die damit aber auch potenziell Verantwortung übernimmt, falls z. B. der Sturz eines Radfahrers durch einen solchen Einbau verursacht werden sollte. Dieses Risiko lag also bei einem kommunalen Akteur, der einer rechtlichen Handlungslogik folgt. Das Reallabor steckte dadurch in einer Zwickmühle: Um die Wirkung der Einbauten zu testen, musste schon vorher deren Wirkung bekannt sein und durch das Reallabor nachgewiesen werden. Die Abklärung, wie die Haftungsfrage geregelt werden kann, stellte einen langwierigen Prozess dar.

Im BaWü-Lab SRB stellte sich vor Projektbeginn die Frage, inwiefern Realexperimente in Schulen oder in deren direkter Umgebung realisiert werden können. Schulen stellen einen sensiblen Kontext dar, da die Sicherheit der Kinder und Jugendlichen höchste Priorität genießt. Temporäre physische Interventionen (ähnlich wie Parklets) sind an Schulen nicht denkbar, da sich die Kinder verletzen könnten. Im Falle eines Unfalls wären die Schulleitung und der Schulträger (die Kommune) haftbar, nicht jedoch die Wissenschaftler(innen). Das heißt, die Schule würde im Sinne einer rechtlichen Handlungslogik den Schaden tragen müssen, während die Wissenschaft durch den potenziellen Erkenntnisgewinn den Nutzen aus dem Realexperiment ziehen würde. Das Risiko läge damit ausschließlich bei den Praxispartnern (Schulleitung und Schulträger). Um diesen Konflikt zu vermeiden, wurde im Reallabor ein spezielles methodisches Vorgehen als Alternative zum Realexperiment entwickelt. 
Deswegen sollte bei einer Anpassung der Risikoaufteilung das Verursacherprinzip beachtet werden, indem möglichst diejenigen Akteure, die ein Risiko verursachen, dieses auch tragen. Wenn eine Anwendung des Verursacherprinzips nicht möglich ist, sollte der Akteur, der das Risiko tragen müsste, diesem zustimmen können, so dass eine bewusste Übernahme stattfinden könnte. Dabei sind die Grenzen der Möglichkeit zur Übernahme von Risiken, die sich bei den verschiedenen Akteuren aufgrund ihrer jeweiligen Handlungslogiken ergeben, zu berücksichtigen. Weiterhin bietet sich der Risikotransfer zu denjenigen Akteuren zum Beispiel in einem Reallabor an, die eine höhere Risikoaffinität besitzen. So kann in einem Reallabor für bestimmte risikobehaftete Realexperimente ein risikoaffiner Akteur die Verantwortung übernehmen und dies in klaren Regelungen zur Verantwortungsübernahme festhalten.

Im BaWü-Lab GO Karlsruhe wurde bei der Öffentlichkeitsarbeit zu den Realexperimenten in den Vordergrund gestellt, dass diese durch Akteure aus der Wissenschaft vorbereitet, umgesetzt und begleitet werden. Damit wurde versucht zu gewährleisten, dass das Risiko der Kritik von Bürger(inne)n an den Realexperimenten sich nicht auf die Kommune, sondern auf die Wissenschaftler(innen) konzentriert. Diese haben die Kritik gesammelt und mussten auf diese auch auf Bürgerveranstaltungen reagieren.

Teile eines Forschungsprojekts, wie zum Beispiel ein bestimmtes Realexperiment, können schließlich von einem Teil der Projektpartner in Abhängigkeit der jeweils wirkenden Handlungslogik als Misserfolg angesehen werden. Dies kann von den Akteuren aus der Wissenschaft berücksichtigt werden, indem entsprechende Vorsorgestrategien entwickelt werden, wie dem von einem Schaden betroffenen Teilsystem vermittelt werden kann, dass für ein anderes Teilsystem derselbe Projektteil positiv verlaufen ist. Auch hier gilt, dass der Blick auf das Projekt als Ganzes eine differenziertere Sicht erlaubt, als wenn die einzelnen Akteure nur auf ihre eigenen Risiken und Chancen fokussieren.

\subsection{Risiken bewusst antizipieren - Prävention und Nachsorge vereinbaren}

Eine bewusste Auseinandersetzung mit verschiedenen Risikokulturen kann den Eintritt von Schäden reduzieren, indem sie ein Risikomanagement ermöglicht. Ein solcher Analyseprozess kann zudem eine positive Teamerfahrung für ein Projekt sein. Aus diesen Gründen ist es wichtig, Risiken bewusst zu antizipieren sowie Prävention und Nachsorge zu vereinbaren.

$\mathrm{Zu}$ relevanten Zeitpunkten im Ablauf eines transdisziplinären (und transformativen) Forschungsprojekts, zum Beispiel bei der konkreten Planung eines Realexperiments, ist es wichtig, das jeweils unterschiedliche Verständnis der Akteure für die Identifikation, Bewertung und Steuerung von Risiken offenzulegen und zu diskutieren. Dies kann beispielsweise im Rahmen eines Workshops geschehen. 
Zudem sind Entscheidungsmechanismen zur Risikosteuerung vorab mit allen Projektpartnern einvernehmlich zu vereinbaren. Zwischen den Akteuren kann es unterschiedliche Präferenzen geben, welche Risikosteuerungsstrategien bevorzugt werden. Risikoaverse Akteure wie Kommunen bevorzugen tendenziell ursachenbezogene Maßnahmen, mit der die Eintrittswahrscheinlichkeit von Schäden reduziert wird, da angestrebt wird, diese wenn immer möglich ganz zu vermeiden. Risikoaffinere Akteure wie zum Beispiel Akteure aus der Wissenschaft berücksichtigen hingegen eher wirkungsbezogene Maßnahmen, die darauf zielen, die Konsequenzen möglicher Schäden zu reduzieren.

Solche unterschiedlichen Präferenzen können zum Beispiel zu Differenzen zwischen Akteuren bezüglich Realexperimenten führen. So können Verwaltungseinheiten eine Nichtgenehmigung von Realexperimenten bevorzugen, wenn sie diese als zu risikoreich einstufen, während die Wissenschaftler(innen) eine Setzung von Maßnahmen zur Risikoreduktion anstreben würden. In solchen Aushandlungsprozessen zwischen den Akteuren ist die unterschiedliche Beurteilung von Schäden zu berücksichtigen. Wissenschaftler(innen) stufen die Nichtumsetzung eines Realexperiments als Schaden ein, da somit die Möglichkeit für Erkenntnisgewinn ausbleibt. Die Verwaltung sieht dagegen den Schaden in der Umsetzung rechtlich unzulässiger Maßnahmen.

Trotz sorgfältiger Planung, Konfliktprävention und Risikomanagement kann es geschehen, dass Realexperimente oder andere Projektteile für die Akteure eines oder mehrerer Teilsysteme zum Misserfolg werden. Was Misserfolg ist beziehungsweise was als Misserfolg empfunden wird, kann dabei je nach Teilsystem sehr unterschiedlich ausfallen. Relevant bei der Bewertung von Erfolg und Misserfolg ist vor allem die Handlungslogik der unterschiedlichen gesellschaftlichen Teilsysteme. So kann beispielsweise eine Intervention im öffentlichen Raum in einem Projekt zwar nicht den von der Stadtverwaltung gewünschten Effekt haben, aber dabei helfen, umfangreiche Daten zu erheben. In einem solchen Fall steht die nach außen als verantwortlich wahrgenommene Verwaltung möglicherweise in der Kritik durch Anwohner(innen) oder die Presse, obwohl durch die Intervention wertvolle Erkenntnisse für Veröffentlichungen oder weitere Interventionen gewonnen wurden. Nur durch einen offenen internen Dialog oder den vorher einkalkulierten Eintritt eines Schadenfalls ist es möglich, anschließend noch ähnliche Aktionen durchzuführen und auch weiterhin vertrauensvoll kooperieren zu können. Umgekehrt kann es auch geschehen, dass eine öffentliche Intervention den von den Teilsystemen Politik und/oder Zivilgesellschaft erwünschten Effekt erzielt, aber keine wissenschaftlichen Erkenntnisse erbringt. In einem solchen Fall führt die Forschung z. B. nicht zu Publikationen, wodurch die beteiligten Wissenschaftler(innen) in die Kritik beispielsweise seitens des Fördergebers (und der anderen Akteure im Teilsystem Wissenschaft) geraten können. Hier könnte die Prävention in einem offenen Dialog über Qualitätskriterien mit dem Fördergeber bestehen. 


\section{Was sonst noch zu sagen wäre ...}

Der vorliegende Beitrag wurde auf Grundlage der Erfahrungen aus verschiedenen BaWü-Labs entwickelt. Während der gemeinsamen Arbeit und der Reflexion über diese Erfahrungen wurde den Autor(inn)en erneut deutlich, dass es in jedem BaWü-Lab wichtig war, Konflikte zwischen unterschiedlichen Akteuren und Akteursgruppen zu vermeiden. Dabei waren weder die Konflikte noch der Umgang damit identisch. Ein Grund für diese Diversität war, dass jedes BaWü-Lab verschiedene Zusammensetzungen von Vertreter(inne)n der beschriebenen Teilsysteme mit ihren jeweiligen Handlungslogiken aufwies. Außerdem hatte jedes BaWü-Lab, bezogen auf die Verteilung von inhaltlichen, finanziellen, organisatorischen, rechtlichen und kommunikativen Verantwortlichkeiten auf die Beteiligten der verschiedenen Teilsysteme, eine eigene Struktur gewählt. Aufgrund der Bandbreite der Erfahrungen aus den BaWü-Labs liefert dieser Beitrag eine recht umfassende Darstellung möglicher Risiken und möglicherweise auftretender Konflikte. Jedoch und trotzdem ist der Beitrag nicht abschließend und erhebt auch keinen Anspruch auf Vollständigkeit, denn jedes transdisziplinäre (und transformative) Forschungsprojekt hat seine eigene Konstellation von Teilsystemen und wird entsprechend seinen eigenen Lösungsweg hinsichtlich potenzieller Risiken und Konflikte finden müssen.

Gemeinsam war den BaWü-Labs jedoch ein ständig anhaltender dynamischer Prozess, der kaum vorhersehbar oder planbar war. Dieser ging meist einher mit Änderungen von Bedingungen und Verschiebungen in der Ausgangslage. So zogen beispielsweise politische Wahlen Veränderungen der Rahmenbedingungen nach sich. Dies konnte zu personellen Änderungen oder zu inhaltlichen Neuausrichtungen einzelner Vertreter(innen) der Teilsysteme führen. Mit Blick darauf wird von den Autor(inn)en ein wiederholtes Überprüfen von Risiken und Konflikten auch während der Projektlaufzeit empfohlen.

Bei der Betrachtung der Teilsysteme unserer funktionalen Gesellschaft wurde deutlich, dass es sich um ein sehr effizientes - über die Historie hinweg entwickeltes - Gesellschaftssystem handelt. Dieses scheint jedoch eher unflexibel zu sein und im Widerspruch zu Innovation und vor allem zur Transformation zu stehen. Insofern wird ein großer Mehrwert in der transdisziplinären (und transformativen) Forschung gesehen. Denn erst durch das Ausloten dieser funktionalen ,Grenzen' zwischen den gesellschaftlichen Teilsystemen und das Infragestellen von Setzungen innerhalb dieser ,Grenzen' entstehen Konflikte, aber auch Synergien zwischen den Teilsystemen. Dieser Prozess kann - losgelöst vom eigentlichen Inhalt eines transdisziplinären Projekts - transformativ wirken für ein Teilsystem, indem andere Leitwerte, Leitdifferenzen und Risikokulturen Berücksichtigung finden. Die Herausforderung besteht also darin, diese Chance der 
transdisziplinären (und transformativen) Forschung zu nutzen, gleichzeitig mit den Risiken umzugehen und mögliche Konflikte nicht zu scheuen, sondern diese offen anzusprechen und produktiv anzugehen. Dies soll durch diesen Beitrag unterstützt werden.

\section{Dank}

Ein besonderer Dank der Autor(inn)en geht an Marius Albiez, Monika Bachinger, Richard Beecroft, Tobias Bernecker, Fabian Dembski, Raphael Dietz, Laura Gebhardt, Mike Letzgus, Editha Marquardt, Thomas Potthast, Regina Rhodius und Michael Ruddat für die Bereitstellung von Beispielen aus ihren jeweiligen Reallaboren. Sie danken Marius Albiez, Fabian Dembski, Raphael Dietz, Thorsten Erl und Jan Riel für die Diskussion über eine frühere Textfassung im Rahmen des internen Reviews. Die Autor(inn)en möchten zudem den beiden anonymen externen Gutachter(inne)n für die konkreten Hinweise zur Verbesserung des Textes danken. Schließlich danken sie den beiden Herausgebenden, Rico Defila und Antonietta Di Giulio, für ihre Rückmeldungen zum Text.

\section{Literatur}

Arnold, A., \& Piontek, F. (2018). Zentrale Begriffe im Kontext der Reallaborforschung. In R. Defila \& A. Di Giulio (Hrsg.), Transdisziplinär und transformativ forschen. Eine Methodensammlung (S. 143-154). Wiesbaden: Springer VS. doi: 10.1007/978-3-65821530-9_8.

Baecker, D. (2016). Wirtschaft als funktionales Teilsystem. In A. Maurer (Hrsg.), Handbuch der Wirtschaftssoziologie (S. 163-180). Wiesbaden: Springer VS.

Bechmann, G., \& Stehr, N. (2000). Risikokommunikation und die Risiken der Kommunikation wissenschaftlichen Wissens: Zum gesellschaftlichen Umgang mit Nichtwissen. GAIA, 9 (2), (S. 113-121).

Beecroft, R. (2019). Das „Transformative Projektseminar“ - didaktische Ansätze und methodische Umsetzung. In R. Defila \& A. Di Giulio (Hrsg.), Transdisziplinär und transformativ forschen, Band 2. Eine Methodensammlung (S. 293-337). Wiesbaden: Springer VS.

Bonß, W. (2011). (Un-)Sicherheit in der Moderne. In P. Zoche, S. Kaufman \& R. Haverkamp (Hrsg.), Zivile Sicherheit. Gesellschaftliche Dimensionen gegenwärtiger Sicherheitspolitiken (S. 43-109). Bielefeld: transcript.

Brugger, P. (2009). Der IT Business Case. Wiesbaden: Springer. 
Callies, G.-P. (2006). Systemtheorie: Luhmann/Teubner. In S. Buckel, R. Christensen \& A. Fischer-Lescano (Hrsg.), Neue Theorien des Rechts (S. 57-74). Stuttgart: Lucius \& Lucius.

Crouch, C. (2008). Postdemokratie. Frankfurt a. M.: Suhrkamp.

DIN ISO 31000 (2018). Risikomanagement - Leitlinien (ISO 31000:2018). doi: 10.31030/ 2880923.

Douglas, M. (1992). Risk and blame essays in cultural theory. London: Routledge.

Egner, H. (2008). Warum konnte das nicht verhindert werden? Über den (Nicht-)Zusammenhang von wissenschaftlicher Erkenntnis und politischen Entscheidungen. In C. Felgentreff \& T. Glade (Hrsg.), Naturrisiken und Sozialkatastrophen (S. 421-433). Berlin, Heidelberg: Springer.

Fischer, S. (2016). Risiko und Risikokultur - Konzepte für die Beschreibung und Analyse des gesellschaftlichen Umgangs mit Unsicherheit. Zeitschrift für Außen- und Sicherheitspolitik, 9 (2), (S. 191-200).

Hanebuth, A., Lee, R., Meschke, S., \& Nicklas, M. (2015). Schlussbetrachtung. In A. Hanebuth, R. Lee, S. Meschke, \& M. Nicklas (Hrsg.), Forschungskooperationen zwischen Wissenschaft und Praxis. Erkenntnisse und Tipps für das Management (S. 225233). Wiesbaden: Springer Gabler.

Heldrich, A. (2015). Die zivilrechtliche Haftung für Schäden durch wissenschaftliche Betätigung. Ordnung der Wissenschaft, (3), (S. 155-164).

Hill, H. (2016). Innovationsmanagement in der Verwaltung. In Innovativer Staat 2017. Das Jahrbuch für die Verwaltung der Zukunft (S. 66-67). Berlin: Wegweiser Media \& Conferences $\mathrm{GmbH}$.

Hoitsch, H. J., Winter, P., \& Bächle, R. (2005). Risikokultur und risikopolitische Grundsätze: Strukturierungsvorschläge und empirische Ergebnisse. Controlling und Management, 49 (2), (S. 125-133).

Huber, I. (2013). Wissenschaft als Beruf? Das Paradox des heutigen Wissenschaftsverständnisses. Bulletin der Vereinigung der Schweizerischen Hochschuldozierenden, 3 (4), (S. 34-37).

Jahner, S., \& Kremar, H. (2005). Risikokultur als zentraler Erfolgsfaktor für ein ganzheitliches IT Risk Management. IM Information Management \& Consulting, (2), (S. 4754).

Joas, H. (2005). Die kulturellen Werte Europas. Eine Einleitung. In: H. Joas \& K. Wiegandt (Hrsg.), Die kulturellen Werte Europas (S. 11-41). Frankfurt: Fischer Taschenbuch.

Kepplinger, H.-M. (2009). Politikvermittlung. Wiesbaden: Verlag für Sozialwissenschaften. 
Köglberger, K., Dietz, R., Eller, C., Piontek, F. M., Albiez, M., \& Potthast T. (2019). Schutz in der Exposition, Schutz für die Exposition - Wie man in transdisziplinären und transformativen Forschungsformaten mit Ungewohntem und erhöhter Aufmerksamkeit umgeht. In R. Defila \& A. Di Giulio (Hrsg.), Transdisziplinär und transformativ forschen, Band 2. Eine Methodensammlung (S. 93-138). Wiesbaden: Springer VS.

Kromschröder, B. (2003). Risiko - Risikoforschung - Risikokommunikation. In J. Geiss, D. Wortmann \& F. Zuber (Hrsg.), Nachhaltige Entwicklung - Strategie für das 21. Jahrhundert? (S. 123-142). Wiesbaden: VS Verlag für Sozialwissenschaften.

Link, P. (2001). Risikomanagement in Innovationskooperationen. Ein Ansatz zur fairen Aufteilung von Chancen und Risiken. Dissertation, ETH Zürich.

Luhmann, N. (1984). Die Wirtschaft der Gesellschaft als autopoietisches System. Zeitschrift für Soziologie, 13 (4), (S. 308-327).

Luhmann, N. (1988). Die Wirtschaft der Gesellschaft. Frankfurt a. M.: Suhrkamp.

Motel, J., \& Richter, M. (2016). Risikomanagement in einer Bundesbehörde. VM Verwaltung \& Management, 22 (2), (S. 73-82).

Möller, H., \& Bruck, E. (1980). Kommentar zum Versicherungsvertragsgesetz und zu den Allgemeinen Versicherungsbedingungen unter Einschluss des Versicherungsvermittlungsrechtes. Berlin: De Gruyter.

Puttrowait, E., Dietz, R., Gantert, M., \& Heynold, J. (2018). Der Weg zum Realexperiment - Schlüsselakteure identifizieren, Kooperationsstrukturen aufbauen, Projektideen auswählen. In R. Defila \& A. Di Giulio (Hrsg.), Transdisziplinär und transformativ forschen. Eine Methodensammlung (S. 195-232). Wiesbaden: Springer VS.

Rayner, S. (1992). Cultural Theory and risk analysis. In S. Krimsky \& D. Golding (Hrsg.), Social theories of risk (S. 83-115). Santa Barbara: Praeger Publishers.

Reichel, A. (2012). Die Zivilgesellschaft der Gesellschaft? Systemtheoretische Beobachtungen eines unruhigen Funktionssystems. In S. Jansen, E. Schröter \& N. Stehr (Hrsg.), Bürger. Macht. Staat? - Neue Formen der gesellschaftlichen Teilhabe, Teilnahme und Arbeitsteilung (S. 53-73). Wiesbaden: Springer Fachmedien.

Renn, O. (2008). Concepts of risk: an interdisciplinary review part 1 - disciplinary risk concepts. GAIA, 17 (1), (S. 50-66).

RLSS (Reallabor Space Sharing, ABK Stuttgart) (Hrsg.). (2018). One Space Fits All Space Sharing Report. Erfahrungsbericht und Empfehlungen aus zwei Jahren Betriebserfahrung des Reallabor Space Sharing-Pilotprojekts. Staatliche Akademie der Bildenden Künste Stuttgart.

Rudzio, W. (2015). Das politische System der Bundesrepublik Deutschland. Wiesbaden: Springer Verlag.

Schimank, U. (2007). Theorien gesellschaftlicher Differenzierung. Wiesbaden: VS Verlag für Sozialwissenschaften. 
Schimank, U. (2012). Wissenschaft als gesellschaftliches Teilsystem. In S. Maasen, M. Kaiser, M. Reinhart \& B. Sutter (Hrsg.), Handbuch Wissenschaftssoziologie (S. 113-123). Wiesbaden: Springer Fachmedien.

Schmitz, T., \& Wehrheim, M. (2006). Risikomanagement. Grundlagen-Theorie-Praxis. Stuttgart: Kohlhammer.

Schneidewind, U., \& Singer-Brodowski, M. (2014). Transformative Wissenschaft: Klimawandel im deutschen Wissenschafts- und Hochschulsystem. Marburg: Metropolis.

Trenks, H., Waitz, C., Meyer-Soylu, S., \& Parodi, O. (2018). Mit einer Realexperimentreihe Impulse für soziale Innovationen setzen - Realexperimente initiieren, begleiten und beforschen. In R. Defila \& A. Di Giulio (Hrsg.), Transdisziplinär und transformativ forschen. Eine Methodensammlung (S. 233-268). Wiesbaden: Springer VS.

VDI 4006 Blatt 1 (2015). Menschliche Zuverlässigkeit - Ergonomische Forderungen und Methoden der Bewertung. VDI Richtlinie, hrsg. vom Verein Deutscher Ingenieure e. V. Düsseldorf.

Weißensteiner, C. (2014). Reputation als Risikofaktor in technologieorientierten Unternehmen. Status Quo - Reputationstreiber - Bewertungsmodell. Wiesbaden: Springer Fachmedien.

Open Access Dieses Kapitel wird unter der Creative Commons Namensnennung 4.0 International Lizenz (http://creativecommons.org/licenses/by/4.0/deed.de) veröffentlicht, welche die Nutzung, Vervielfältigung, Bearbeitung, Verbreitung und Wiedergabe in jeglichem Medium und Format erlaubt, sofern Sie den/die ursprünglichen Autor(en) und die Quelle ordnungsgemäß nennen, einen Link zur Creative Commons Lizenz beifügen und angeben, ob Änderungen vorgenommen wurden.

Die in diesem Kapitel enthaltenen Bilder und sonstiges Drittmaterial unterliegen ebenfalls der genannten Creative Commons Lizenz, sofern sich aus der Abbildungslegende nichts anderes ergibt. Sofern das betreffende Material nicht unter der genannten Creative Commons Lizenz steht und die betreffende Handlung nicht nach gesetzlichen Vorschriften erlaubt ist, ist für die oben aufgeführten Weiterverwendungen des Materials die Einwilligung des jeweiligen Rechteinhabers einzuholen.

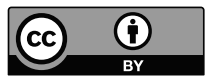

